\title{
A MINIMIZING MOVEMENT APPROACH TO A CLASS OF SCALAR REACTION-DIFFUSION EQUATIONS
}

\author{
Florentine Catharina Fleißner*
}

\begin{abstract}
The purpose of this paper is to introduce a Minimizing Movement approach to scalar reaction-diffusion equations of the form

$$
\partial_{t} u=\Lambda \cdot \operatorname{div}\left[u\left(\nabla F^{\prime}(u)+\nabla V\right)\right]-\Sigma \cdot\left(F^{\prime}(u)+V\right) u, \quad \text { in }(0,+\infty) \times \Omega,
$$
\end{abstract}

with parameters $\Lambda, \Sigma>0$ and no-flux boundary condition

$$
u\left(\nabla F^{\prime}(u)+\nabla V\right) \cdot \mathrm{n}=0, \quad \text { on }(0,+\infty) \times \partial \Omega,
$$

which is built on their gradient-flow-like structure in the space $\mathcal{M}(\bar{\Omega})$ of finite nonnegative Radon measures on $\bar{\Omega} \subset \mathbb{R}^{d}$, endowed with the recently introduced Hellinger-Kantorovich distance $\mathrm{H}_{\Lambda, \Sigma}$. It is proved that, under natural general assumptions on $F:[0,+\infty) \rightarrow \mathbb{R}$ and $V: \bar{\Omega} \rightarrow \mathbb{R}$, the Minimizing Movement scheme

$$
\mu_{\tau}^{0}:=u_{0} \mathscr{L}^{d} \in \mathcal{M}(\bar{\Omega}), \quad \mu_{\tau}^{n} \text { is a minimizer for } \mathcal{E}(\cdot)+\frac{1}{2 \tau} \mathrm{K}_{\Lambda, \Sigma}\left(\cdot, \mu_{\tau}^{n-1}\right)^{2}, n \in \mathbb{N},
$$

for

$$
\mathcal{E}: \mathcal{M}(\bar{\Omega}) \rightarrow(-\infty,+\infty], \mathcal{E}(\mu):= \begin{cases}\int_{\Omega}[F(u(x))+V(x) u(x)] \mathrm{d} x & \text { if } \mu=u \mathscr{L}^{d}, \\ +\infty & \text { else, }\end{cases}
$$

yields weak solutions to the above equation as the discrete time step size $\tau \downarrow 0$. Moreover, a superdifferentiability property of the Hellinger-Kantorovich distance $\mathrm{HK}_{\Lambda, \Sigma}$, which will play an important role in this context, is established in the general setting of a separable Hilbert space; that result will constitute a starting point for the study of the differentiability of $\mathrm{H}_{\Lambda, \Sigma}$ along absolutely continuous curves which will be carried out in a subsequent paper.

Mathematics Subject Classification. 35K57, 35K20, 35K55, 49M25, 47J25, 47J30, 28A33, 54E35, 46G99, 49Q20.

Received February 11, 2020. Accepted December 15, 2020.

Keywords and phrases: Optimal transport, gradient flows, Minimizing Movements, reactiondiffusion equations, HellingerKantorovich distance.

Technische Universität München, München, Germany.

* Corresponding author: fleissne@ma.tum.de 


\section{INTRODUCTION}

At the beginning of the 90's, Ennio De Giorgi introduced the concept of Minimizing Movement as "natural meeting point" of many evolution problems from different research fields in mathematics [7]. He got his inspiration from the paper [1] by Almgren, Taylor and Wang. The concept involves the recursive minimization

$$
u_{\tau}^{0}:=u_{0} \in \mathscr{S}, \quad u_{\tau}^{n} \text { is a minimizer for } \Phi\left(\tau, u_{\tau}^{n-1}, \cdot\right), n \in \mathbb{N},
$$

of a given functional $\Phi:(0,1) \times \mathscr{S} \times \mathscr{S} \rightarrow[-\infty,+\infty]$ on a topological space $(\mathscr{S}, \sigma)$. The parameter $\tau>0$ plays the role of discrete time step size. If a sequence $\left(u_{\tau}^{n}\right)_{n \in \mathbb{N}}$ satisfies (1.1), we call the corresponding piecewise constant interpolation $u_{\tau}:[0,+\infty) \rightarrow \mathscr{S}, u_{\tau}(0)=u_{0}, u_{\tau}(t) \equiv u_{\tau}^{n}$ for $t \in((n-1) \tau, n \tau](n \in \mathbb{N})$, a discrete solution. The concept's purpose is to study the limit curves as $\tau \downarrow 0$.

Definition 1.1 ((Generalized) Minimizing Movement [7]). A curve $u:[0,+\infty) \rightarrow \mathscr{S}$ is called Minimizing Movement for $\Phi$ with initial datum $u_{0}$ (short $u \in \operatorname{MM}\left(\Phi ; u_{0}\right)$ ) if there exist discrete solutions $u_{\tau}$ to (1.1) (for $\tau>0$ in a right neighbourhood of 0 ) such that $u_{\tau}^{0}=u_{0}=u(0)$ and $u_{\tau}(t) \stackrel{\sigma}{\rightarrow} u(t)$ for all $t>0$ as $\tau \downarrow 0$. A curve $u:[0,+\infty) \rightarrow \mathscr{S}$ is called Generalized Minimizing Movement for $\Phi$ with initial datum $u_{0}$ (short $\left.u \in \operatorname{GMM}\left(\Phi ; u_{0}\right)\right)$ if there exist a subsequence of time steps $\left(\tau_{k}\right)_{k \in \mathbb{N}}, \tau_{k} \downarrow 0$, and discrete solutions $u_{\tau_{k}}$ to $(1.1)$ such that $u_{\tau_{k}}^{0}=u_{0}=u(0)$ and $u_{\tau_{k}}(t) \stackrel{\sigma}{\rightarrow} u(t)$ for all $t>0$ as $k \rightarrow \infty$.

Example 1.2 (Gradient flows in finite dimensional Euclidean space). Let $\mathbb{H}$ be a finite dimensional Euclidean space with norm $|\cdot|$ and $\mathcal{E} \in \mathrm{C}^{1}(\mathbb{H})$ satisfy the quadratic lower bound

$$
\exists A, B>0: \quad \mathcal{E}(x) \geq-A-B|x|^{2} \quad \text { for all } x \in \mathbb{H} .
$$

We apply the Minimizing Movement scheme (1.1) to $\Phi(\tau, v, x):=\mathcal{E}(x)+\frac{1}{2 \tau}|x-v|^{2}$. The necessary condition of first order leads to a discrete version of the classical gradient flow equation

$$
u^{\prime}(t)=-\nabla \mathcal{E}(u(t)), \quad t \geq 0,
$$

and indeed, it is not difficult to see that every $u \in \operatorname{GMM}\left(\Phi ; u_{0}\right)$ (which is a nonempty set) is a solution to (1.3) with initial datum $u_{0} \in \mathbb{H}$. The statement also holds good if we replace $\mathcal{E}$ by $\mathcal{E}_{\tau}$ in $\Phi$, with $\mathcal{E}_{\tau}: \mathbb{H} \rightarrow \mathbb{R}$ converging to $\mathcal{E}$ in the Lipschitz semi-norm as $\tau \downarrow 0$. Conversely, for every solution $u \in \mathrm{C}^{1}([0,+\infty) ; \mathbb{H})$ to (1.3) there exist functions $\mathcal{E}_{\tau}: \mathbb{H} \rightarrow \mathbb{R}(\tau>0)$ such that $\operatorname{Lip}\left[\mathcal{E}_{\tau}-\mathcal{E}\right] \rightarrow 0$ as $\tau \downarrow 0$ and $\operatorname{MM}(\Phi ; u(0))=\{u\}=\operatorname{GMM}(\Phi ; u(0))$ for $\Phi(\tau, v, x):=\mathcal{E}_{\tau}(x)+\frac{1}{2 \tau}|x-v|^{2}$, see [12]. This gives a full characterization of solutions to (1.3) as (Generalized) Minimizing Movements.

De Giorgi's concept of Minimizing Movements has a wide range of applications in analysis, geometry, physics and numerical analysis, and we refer to $[1,3,7,25,27,28]$ for more examples. In this paper the focus will be on the Minimizing Movement approach to gradient flows.

The Minimizing Movement scheme from Example 1.2 can be adapted for a general metric and non-smooth setting: Let a functional $\mathcal{E}: \mathscr{S} \rightarrow(-\infty,+\infty]$ on a complete metric space $(\mathscr{S}, d)$ be given and apply $(1.1)$ to

$$
\Phi(\tau, v, x):=\mathcal{E}(x)+\frac{1}{2 \tau} d(x, v)^{2} .
$$

It is proved in [2] that, under natural coercivity assumptions, the set $\operatorname{GMM}\left(\Phi ; u_{0}\right)$ is nonempty for every initial datum $u_{0} \in\{\mathcal{E}<+\infty\}$ and the Generalized Minimizing Movements are locally absolutely continuous curves satisfying the energy dissipation inequality

$$
\mathcal{E}(u(0))-\mathcal{E}(u(t)) \geq \frac{1}{2} \int_{0}^{t}\left|\partial^{-} \mathcal{E}\right|(u(r))^{2} \mathrm{~d} r+\frac{1}{2} \int_{0}^{t}\left|u^{\prime}\right|(r)^{2} \mathrm{~d} r
$$


for all $t>0$, with $\left|\partial^{-} \mathcal{E}\right|$ denoting the relaxed slope of $\mathcal{E}$ (which can be viewed as a weak counterpart of the modulus of the gradient) and $\left|u^{\prime}\right|$ the metric derivative of $u$ (see ([2], Chaps. 1 and 2) for the corresponding definitions or Section 4.3 in this paper for a brief overview). Under the additional assumption that the relaxed slope satisfies a kind of metric chain rule, equality can be proved in (1.5), see [2]. The characterization of curves via such energy dissipation (in)equality corresponds with the notion of gradient flows in metric spaces which goes back to $[8,9,24]$. It is equivalent to $(1.3)$ if $\mathcal{E} \in \mathrm{C}^{1}(\mathbb{H})$ and $\mathbb{H}$ is a finite dimensional Euclidean space. We refer to $[10]$ for further developments of the theory.

Example 1.2 and the results in metric spaces motivate us to study a Minimizing Movement approach whenever gradient-flow-like structures are discovered and justify the interpretation of dynamics governed by an evolution equation as a gradient flow (for an energy functional on a metric space) if the corresponding (Generalized) Minimizing Movements are solutions to the evolution equation.

The following Minimizing Movement approach to scalar diffusion equations of the form

$$
\partial_{t} u(t, x)=\operatorname{div}\left[u(t, x)\left(\nabla F^{\prime}(u(t, x))+\nabla V(x)\right)\right], \quad t>0, x \in \mathbb{R}^{d},
$$

has its origin in the papers $[16,17]$ by Jordan, Kinderlehrer and Otto, was examined by Ambrosio, Gigli and Savaré in [2] and has been taken in many applications (see e.g. ([2], Chapt. 11) and the references therein): The space $\mathcal{P}_{2}\left(\mathbb{R}^{d}\right)$ of Borel probability measures with finite second order moments $\left(\right.$ i.e. $\left.\int_{\mathbb{R}^{d}}|x|^{2} \mathrm{~d} \mu<+\infty\right)$ is endowed with the quadratic Wasserstein distance $\mathcal{W}_{2}$,

$$
\mathcal{W}_{2}\left(\mu_{1}, \mu_{2}\right)^{2}:=\min _{\gamma \in P\left(\mu_{1}, \mu_{2}\right)} \int_{\mathbb{R}^{d} \times \mathbb{R}^{d}}|x-y|^{2} \mathrm{~d} \gamma, \quad \mu_{i} \in \mathcal{P}_{2}\left(\mathbb{R}^{d}\right)
$$

with $P\left(\mu_{1}, \mu_{2}\right)$ being the set of Borel probability measures on $\mathbb{R}^{d} \times \mathbb{R}^{d}$ whose first and second marginals coincide with $\mu_{1}$ and $\mu_{2}$ respectively (see e.g. [30,31] for a detailed account of the theory of Optimal Transport and Wasserstein distances). The functional $\mathcal{E}: \mathcal{P}_{2}\left(\mathbb{R}^{d}\right) \rightarrow(-\infty,+\infty]$,

$$
\mathcal{E}(\mu):= \begin{cases}\int_{\mathbb{R}^{d}}[F(u(x))+V(x) u(x)] \mathrm{d} x & \text { if } \mu=u \mathscr{L}^{d}\left(\mathscr{L}^{d} \text { d-dimensional Lebesgue measure }\right) \\ +\infty & \text { else },\end{cases}
$$

is defined on $\left(\mathcal{P}_{2}\left(\mathbb{R}^{d}\right), \mathcal{W}_{2}\right)$ and $\Phi$ is defined according to (1.4). Under suitable assumptions on $F:[0,+\infty) \rightarrow \mathbb{R}$ and $V: \mathbb{R}^{d} \rightarrow \mathbb{R}$, the corresponding Minimizing Movement scheme (often referred to as 'JKO-scheme' in the literature) yields weak solutions to (1.6) (cf. the exemplary proof for $F(u)=u \log u$ and nonnegative $V \in$ $\mathrm{C}^{\infty}\left(\mathbb{R}^{d}\right)$ in [17] and Chaps. 10.1, 10.4 and 11.1.3 in [2]): Such setting typically includes the assumptions that $F$ is convex, continuous with $F(0)=0$, differentiable in $(0,+\infty)$, has superlinear growth and is bounded from below by $s \mapsto-C s^{\lambda}$ for some $\lambda>\frac{d}{d+2}, C>0$, as well as local Lipschitz continuity and nonnegativity of $V$.

This paper concerns scalar reaction-diffusion equations of the form

$$
\partial_{t} u(t, x)=\Lambda \cdot \operatorname{div}\left[u(t, x)\left(\nabla F^{\prime}(u(t, x))+\nabla V(x)\right)\right]-\Sigma \cdot\left(F^{\prime}(u(t, x))+V(x)\right) u(t, x), \quad t>0, x \in \Omega \subset \mathbb{R}^{d},
$$

with fixed parameters $\Lambda>0$ and $\Sigma>0$ (with which the diffusion part and the reaction part respectively are weighted). Moreover, the reaction part is governed by the growth / shrinkage rate $\mathfrak{G}(x, u):=-\left(F^{\prime}(u)+V(x)\right)$ (with $F:[0,+\infty) \rightarrow \mathbb{R}, V: \Omega \rightarrow \mathbb{R}$ ) which also affects the diffusion part since according to (1.9), diffusion occurs along the gradient $\nabla \mathfrak{G}(x, u(x))$ from regions of lower to higher growth rate / from regions of higher to lower shrinkage rate. Equation (1.9) seems a likely model for describing the evolution in time of the density $u$ of some biological, ecological, economic, ... quantity in various cases in which there is not only diffusion but also generation and annihilation of mass and in which the motion of the particles, members of the species, ... is influenced by their tendency to move towards regions with the most "favourable" conditions (see e.g. ([19], Sect. 4), [18, 20] and the references therein for applications). Reaction-diffusion equations of the above form 
are closely related to a distance on the space of finite nonnegative Radon measures which has been recently introduced independently of each other by three different teams $[5,6,19,22,23]$. We follow the presentation of the distance by Liero, Mielke and Savaré $[22,23]$ who named it Hellinger-Kantorovich distance.

\subsection{The Hellinger-Kantorovich distance}

Let $(X, \mathrm{~d})$ be a Polish space (i.e. a complete separable metric space) and let $\mathcal{M}(X)$ be the space of finite nonnegative Radon measures on it. The class of Hellinger-Kantorovich distances $H_{\Lambda, \Sigma}(\Lambda, \Sigma>0)$ can be characterized by the Logarithmic Entropy-Transport problems

$\operatorname{LT}_{\Lambda, \Sigma}\left(\mu_{1}, \mu_{2}\right):=\min \left\{\sum_{i=1}^{2} \frac{4}{\Sigma} \int_{X}\left(\sigma_{i} \log \sigma_{i}-\sigma_{i}+1\right) \mathrm{d} \mu_{i}+\int_{X \times X} \mathrm{c}_{\Lambda, \Sigma}\left(\mathrm{d}\left(x_{1}, x_{2}\right)\right) \mathrm{d} \gamma: \gamma \in \mathcal{M}(X \times X), \gamma_{i} \ll \mu_{i}\right\}$,

for $\mu_{i} \in \mathcal{M}(X)$, with

$$
\sigma_{i}:=\frac{\mathrm{d} \gamma_{i}}{\mathrm{~d} \mu_{i}}\left(\gamma_{i} \text { i-th marginal of } \gamma\right), \quad \mathrm{c}_{\Lambda, \Sigma}(\mathrm{d}):= \begin{cases}-\frac{8}{\Sigma} \log (\cos (\sqrt{\Sigma /(4 \Lambda)} \mathrm{d})) & \text { if } \mathrm{d}<\pi \sqrt{\Lambda / \Sigma} \\ +\infty & \text { if } \mathrm{d} \geq \pi \sqrt{\Lambda / \Sigma}\end{cases}
$$

An optimal plan $\gamma$ (which exists by [23], Thm. 3.3) describes an optimal way of converting $\mu_{1}$ into $\mu_{2}$ (possibly having different total mass) by means of transport and creation / annihilation of mass, in view of the transportation cost function $\mathrm{c}_{\Lambda, \Sigma}(\mathrm{d})$ and the entropy cost functions $\frac{4}{\Sigma}\left(\sigma_{i} \log \sigma_{i}-\sigma_{i}+1\right)$. The bigger the parameter $\Lambda>0$ is (for the same $\Sigma>0$ ), the more the system favours transport. The bigger $\Sigma>0$ is (for the same $\Lambda>0$ ), the more the system favours creation and annihilation of mass.

Proposition 1.3 (cf. [23], Cor. 7.14, Thms. 7.15, 7.17, 7.20, Lem. 7.8 and [22], Sect. 3). For all $\Lambda, \Sigma>0$ :

(1) $\mathrm{HK}_{\Lambda, \Sigma}: \mathcal{M}(X) \times \mathcal{M}(X) \rightarrow[0,+\infty), \mathrm{HK}_{\Lambda, \Sigma}\left(\mu_{1}, \mu_{2}\right):=\sqrt{\mathbb{E T}_{\Lambda, \Sigma}\left(\mu_{1}, \mu_{2}\right)}$, is a distance on $\mathcal{M}(X)$.

(2) $\mathrm{H}_{\Lambda, \Sigma}$ metrizes the weak topology on $\mathcal{M}(X)$ in duality with continuous and bounded functions $\phi: X \rightarrow \mathbb{R}$ (short $\left.\phi \in \mathrm{C}_{b}^{0}(X)\right)$, i.e.

$$
\lim _{n \rightarrow \infty} \mathrm{HK}_{\Lambda, \Sigma}\left(\mu_{n}, \mu\right)=0 \quad \text { if and only if } \quad \lim _{n \rightarrow \infty} \int_{X} \phi \mathrm{d} \mu_{n}=\int_{X} \phi \mathrm{d} \mu \text { for all } \phi \in \mathrm{C}_{b}^{0}(X) \text {. }
$$

(3) $\left(\mathcal{M}(X), \mathrm{H}_{\Lambda, \Sigma}\right)$ is a complete metric space.

(4) Let $\eta_{0}$ denote the null measure. For all $\mu \in \mathcal{M}(X)$ :

$$
\mathrm{H}_{\Lambda, \Sigma}\left(\mu, \eta_{0}\right)^{2}=\frac{4}{\Sigma} \mu(X)
$$

If $X=\mathbb{R}^{d}$ or $X$ is a compact, convex subset of $\mathbb{R}^{d}$ and $\mathrm{d}$ is induced by the usual norm, then a representation formula à la Benamou-Brenier can be proved for $\mathrm{H}_{\Lambda, \Sigma}$ (see ([23], Thms. 8.18, 8.20; [22], Thm. 3.6(v))):

$$
\mathrm{H}_{\Lambda, \Sigma}\left(\mu_{1}, \mu_{2}\right)^{2}=\inf \left\{\int_{0}^{1} \int_{X}\left(\Lambda|\nabla \xi(t, x)|^{2}+\Sigma|\xi(t, x)|^{2}\right) \mathrm{d} \mu_{t}(x) \mathrm{d} t: \mu_{1} \stackrel{(\mu, \xi)}{\rightsquigarrow} \mu_{2}\right\}
$$

where $\mu_{1} \stackrel{(\mu, \xi)}{\leftrightarrow} \mu_{2}$ means that $\mu:[0,1] \rightarrow \mathcal{M}(X)$ is a continuous curve connecting $\mu(0)=\mu_{1}$ and $\mu(1)=\mu_{2}$ and satisfying the continuity equation with reaction $\partial_{t} \mu_{t}=-\Lambda \operatorname{div}\left(\mu_{t} \nabla \xi_{t}\right)+\Sigma \mu_{t} \xi_{t}$, governed by $\xi:(0,1) \times X \rightarrow \mathbb{R}$ with $\xi(t, \cdot)$ Lipschitz continuous and bounded for all $t \in(0,1)$, in duality with $\mathrm{C}^{\infty}$-functions with compact 
support in $(0.1) \times X$, i.e.

$$
\int_{0}^{1} \int_{X}\left(\partial_{t} \psi(t, x)+\Lambda \nabla \psi(t, x) \cdot \nabla \xi(t, x)+\Sigma \psi(t, x) \xi(t, x)\right) \mathrm{d} \mu_{t}(x) \mathrm{d} t=0 \quad \text { for all } \psi \in \mathrm{C}_{c}^{\infty}((0,1) \times X) .
$$

Hence, on the set $\left\{\mu \in \mathcal{M}(X): \mu=u \mathscr{L}^{d}\right\}$ of absolutely continuous Radon measures with respect to the Lebesgue measure, $\mathrm{H}_{\Lambda, \Sigma}$ can be identified with the dissipation distance $\mathcal{D}_{\mathbb{K}_{\Lambda, \Sigma}}$,

$$
\mathcal{D}_{\mathbb{K}_{\Lambda, \Sigma}}\left(u_{1}, u_{2}\right)^{2}:=\inf \left\{\int_{0}^{1}<\xi(t), \mathbb{K}_{\Lambda, \Sigma}\left(u_{t}\right) \xi(t)>\mathrm{d} t: \partial_{t} u_{t}=\mathbb{K}_{\Lambda, \Sigma}\left(u_{t}\right) \xi(t), u_{1} \stackrel{(u, \xi)}{\rightsquigarrow} u_{2}\right\},
$$

generated by the Onsager operator $\mathbb{K}_{\Lambda, \Sigma}(u) \xi:=-\Lambda \operatorname{div}(u \nabla \xi)+\Sigma u \xi$, which suggests a gradient-flow-like structure of (1.9) associated with the energy functional $\mathcal{E}: \mathcal{M}(\Omega) \rightarrow(-\infty,+\infty]$,

$$
\mathcal{E}(\mu):= \begin{cases}\int_{\Omega}[F(u(x))+V(x) u(x)] \mathrm{d} x & \text { if } \mu=u \mathscr{L}^{d}, \\ +\infty & \text { else }\end{cases}
$$

on $\left(\mathcal{M}(\Omega), \mathrm{H}_{\Lambda, \Sigma}\right)$ (for details we refer to Sect. 2 in [22], [26], Sect. 3.2 in [19], and Otto's Riemannian formalism for $\left(\mathcal{P}_{2}\left(\mathbb{R}^{d}\right), \mathcal{W}_{2}\right)$ in $\left.[29]\right)$.

To handle such equation (in a weak form), Gallouët and Monsaigeon proposed a 'JKO splitting scheme' in [14], in which one step $\mu_{\tau}^{n} \curvearrowright \mu_{\tau}^{n+1}$ consists of two substeps

$$
\begin{aligned}
\mu_{\tau}^{n+1 / 2} & \text { is a minimizer for } \mathcal{E}(\cdot)+\frac{1}{2 \tau} \mathcal{W}_{2}\left(\cdot, \mu_{\tau}^{n}\right)^{2}, \\
\mu_{\tau}^{n+1} & \text { is a minimizer for } \mathcal{E}(\cdot)+\frac{1}{2 \tau} \operatorname{He}\left(\cdot, \mu_{\tau}^{n+1 / 2}\right)^{2}
\end{aligned}
$$

(for $\Lambda=\Sigma=1$ ), and which is justified by the interpretation of the Hellinger-Kantorovich distance as infimal convolution of the Kantorovich-Wasserstein distance $\mathcal{W}_{2}$ and the Hellinger-Kakutani/Fisher-Rao distance $\mathrm{He}$ (cf. (1.14) and (1.16)). In this paper, we will work directly with the Hellinger-Kantorovich distance $\mathrm{HK}_{\Lambda, \Sigma}$ and take the 'natural' Minimizing Movement approach to (1.9), associated with

$$
\Phi(\tau, \mu, \nu):=\mathcal{E}(\nu)+\frac{1}{2 \tau} \mathrm{K}_{\Lambda, \Sigma}(\nu, \mu)^{2} .
$$

Before presenting our results, we would like to mention [4] in which such approach has been taken for a particular equation of Hele-Shaw type, which serves as a model for tumour growth. The considerations therein are based on the special structure of the corresponding energy functional

$$
\mathcal{E}(\mu):= \begin{cases}-c \mu(\Omega) & \text { if } \mu=u \mathscr{L}^{d} \text { and } u \leq 1, \\ +\infty & \text { else }\end{cases}
$$

(for $c>0$ ) and do not overlap with our analysis.

\subsection{Our Minimizing Movement approach}

Let $\Omega$ be an open, bounded, convex subset of $\mathbb{R}^{d}$ with $\mathrm{C}^{1}$-boundary $\partial \Omega$, and for $\Lambda, \Sigma>0$, let the space $\mathcal{M}(\bar{\Omega})$ of finite nonnegative Radon measures on its closure be endowed with the Hellinger-Kantorovich distance $\mathrm{HK}_{\Lambda, \Sigma}$, i.e. set $X:=\bar{\Omega}$ and $\mathrm{d}\left(x_{1}, x_{2}\right):=\left|x_{1}-x_{2}\right|$ (induced by the usual norm $|\cdot|$ on $\left.\mathbb{R}^{d}\right)$ in (1.10) and Proposition 1.3. 
We apply the Minimizing Movement scheme (1.1) to

$$
\begin{gathered}
\Phi(\tau, \mu, \nu):=\mathcal{E}(\nu)+\frac{1}{2 \tau} \mathrm{H}_{\Lambda, \Sigma}(\nu, \mu)^{2}, \\
\mathcal{E}: \mathcal{M}(\bar{\Omega}) \rightarrow(-\infty,+\infty], \quad \mathcal{E}:=\mathcal{F}+\mathcal{V}, \quad \mathcal{F}(\mu):=\left\{\begin{array}{ll}
\int_{\Omega} F(u(x)) \mathrm{d} x & \text { if } \mu=u \mathscr{L}^{d} \\
+\infty & \text { else }
\end{array}, \quad \mathcal{V}(\mu):=\int_{\bar{\Omega}} V(x) \mathrm{d} \mu,\right.
\end{gathered}
$$

where $\mu=u \mathscr{L}^{d}$ means that $u: \Omega \rightarrow[0,+\infty)$ is Borel measurable and $\int_{\bar{\Omega}} \phi(x) \mathrm{d} \mu=\int_{\Omega} \phi(x) u(x) \mathrm{d} x$ for all $\phi \in \mathrm{C}_{b}^{0}(\bar{\Omega})$. We prove that, under natural general assumptions on $F:[0,+\infty) \rightarrow \mathbb{R}$ and $V: \bar{\Omega} \rightarrow \mathbb{R}$ (comparable to the typical assumptions in the case of diffusion equations (1.6), see above), the corresponding sets of Generalized Minimizing Movements $\operatorname{GMM}\left(\Phi ; \mu_{0}\right)$ (for initial data $\mu_{0} \in\{\mathcal{E}<+\infty\}$ ) are nonempty and for every $\mu \in \operatorname{GMM}\left(\Phi ; \mu_{0}\right)$ there is $u:[0,+\infty) \times \Omega \rightarrow[0,+\infty)$ such that $\mu(t)=u(t) \mathscr{L}^{d}$ for all $t \geq 0$ and $u$ solves the scalar reaction-diffusion equation

$$
\partial_{t} u(t, x)=\Lambda \cdot \operatorname{div}\left[u(t, x)\left(\nabla F^{\prime}(u(t, x))+\nabla V(x)\right)\right]-\Sigma \cdot\left(F^{\prime}(u(t, x))+V(x)\right) u(t, x), \quad t>0, x \in \Omega,
$$

with no-flux boundary condition

$$
u(t, x)\left(\nabla F^{\prime}(u(t, x))+\nabla V(x)\right) \cdot \mathrm{n}(x)=0, \quad t>0, x \in \partial \Omega,
$$

in a weak form, see Theorem 4.4. Here, $\mathrm{n}$ denotes the outward pointing unit normal vector field along $\partial \Omega$. We discuss our assumptions on $F$ and $V$ in Section 4.1; they are satisfied for example if $V$ is Lipschitz continuous and $F(u)=c_{1} u \log u\left(c_{1}>0\right)$ or $F(u)=-c_{1} u^{q}+c_{2} u^{p}\left(c_{1} \geq 0, c_{2}>0, p>1, q \in(0,1)\right)$, see Example 4.8. The key to proving our result is that we are able to establish a subdifferentiability property of the opposite Hellinger-Kantorovich distance $-\mathrm{K}_{\Lambda, \Sigma}$ along certain directions. We can identify, for $\mu, \nu_{0} \in \mathcal{M}(\bar{\Omega})$ and curves $h \mapsto \nu_{h} \in \mathcal{M}(\bar{\Omega})$ of the form

$$
\nu_{h}:=(I+h v)_{\#}(1+h R)^{2} \nu_{0}
$$

(where $v: \bar{\Omega} \rightarrow \mathbb{R}^{d}, \quad R: \bar{\Omega} \rightarrow \mathbb{R}$ are bounded and the support of $v$ lies in $\Omega$ ), elements of the Fréchet subdifferentials of the mappings

$$
h \mapsto-\frac{1}{2} \mathrm{H}_{\Lambda, \Sigma}\left(\nu_{h}, \mu\right)^{2}
$$

at $h=0$ and, setting $v:=\frac{4 \Lambda}{\Sigma} \nabla \phi, R:=2 \phi$ in (1.23), we can link them to the difference

$$
\int_{\bar{\Omega}} \phi \mathrm{d} \mu-\int_{\bar{\Omega}} \phi \mathrm{d} \nu_{0}
$$

for any $\mathrm{C}^{2}$-function $\phi: \Omega \rightarrow \mathbb{R}$ with compact support in $\Omega$, see Section 3 . Thereby, the possibility of establishing a discrete weak version of (1.21) for discrete solutions to (1.1) opens up. Further crucial points in our proof will be compactness issues, the passage to the limit $\tau \downarrow 0$ in the discrete weak version of (1.21) and the Neumann boundary condition (1.22).

The analysis of the Fréchet subdifferentials of the mappings (1.24) in Section 3 seems of independent interest and will be carried out for general separable Hilbert spaces.

The plan for the paper is as follows. In Section 2, an equivalent characterization of the Hellinger-Kantorovich distance $\mathrm{HK}_{\Lambda, \Sigma}$ will be given, which will be useful for our study of subdifferentiability properties of $-\mathrm{HK}_{\Lambda, \Sigma}$ 
carried out in Section 3. In Section 4, our Minimizing Movement approach to reaction-diffusion equations with no-flux boundary condition will be established. Our assumptions on $F$ and $V$ will be discussed in Section 4.1 and the proof of our main result will be given in Section 4.2. In Section 4.3, we will make some comments and go into future developments, including the case of an unbounded domain, see Example 4.12.

\section{Hellinger-Kantorovich DistanCE AND OPtimal TRANSPORTATiON ON THE CONE}

Let $(X, \mathrm{~d})$ be a Polish space and define the geometric cone $\mathfrak{C}$ on $X$ as the quotient space

$$
\mathfrak{C}:=X \times[0,+\infty) / \sim
$$

with

$$
\left(x_{1}, r_{1}\right) \sim\left(x_{2}, r_{2}\right) \quad \Leftrightarrow \quad r_{1}=r_{2}=0 \text { or } r_{1}=r_{2}, x_{1}=x_{2}
$$

for $x_{i} \in X, r_{i} \in[0,+\infty$ ). The vertex $\mathfrak{o}$ (for $r=0$ ) and $[x, r]$ (for $x \in X$ and $r>0$ ) denote the corresponding equivalence classes, i.e. $\mathfrak{C}=\{[x, r] \mid x \in X, r>0\} \cup\{\mathfrak{o}\}$.

In ([22], Sect. 3) and ([23], Sect. 7), the Logarithmic Entropy-Transport problem (1.10) for $\Lambda, \Sigma>0$ is translated into a problem of optimal transportation on the cone governed by $\mathrm{d}_{\mathfrak{C}, \Lambda, \Sigma}: \mathfrak{C} \times \mathfrak{C} \rightarrow[0,+\infty)$,

$$
\mathrm{d}_{\mathfrak{C}, \Lambda, \Sigma}\left(\left[x_{1}, r_{1}\right],\left[x_{2}, r_{2}\right]\right)^{2}:=\frac{4}{\Sigma}\left(r_{1}^{2}+r_{2}^{2}-2 r_{1} r_{2} \cos \left(\left(\sqrt{\Sigma / 4 \Lambda} \mathrm{d}\left(x_{1}, x_{2}\right)\right) \wedge \pi\right)\right)
$$

(where $\mathfrak{o}$ is identified with $[\bar{x}, 0]$ for some $\bar{x} \in X$ ) which is a distance on $\mathfrak{C}$. The space $\mathcal{M}_{2}(\mathfrak{C})$ of finite nonnegative Radon measures on the cone with finite second order moments, i.e. $\int_{\mathfrak{C}} d_{\mathfrak{C}, \Lambda, \Sigma}([x, r], \mathfrak{o})^{2} \mathrm{~d} \alpha([x, r])<+\infty$, is endowed with an extended quadratic Kantorovich-Wasserstein distance $\mathcal{W}_{\mathfrak{C}, \Lambda, \Sigma}$,

$$
\mathcal{W}_{\mathfrak{C}, \Lambda, \Sigma}\left(\alpha_{1}, \alpha_{2}\right)^{2}:= \begin{cases}\min \left\{\int_{\mathfrak{C} \times \mathfrak{C}} \mathfrak{d}_{\mathfrak{C}, \Lambda, \Sigma}\left(\left[x_{1}, r_{1}\right],\left[x_{2}, r_{2}\right]\right)^{2} \mathrm{~d} \beta \mid \beta \in M\left(\alpha_{1}, \alpha_{2}\right)\right\} & \text { if } \alpha_{1}(\mathfrak{C})=\alpha_{2}(\mathfrak{C}), \\ +\infty & \text { else, }\end{cases}
$$

with $M\left(\alpha_{1}, \alpha_{2}\right)$ being the set of finite nonnegative Radon measures on $\mathfrak{C} \times \mathfrak{C}$ whose first and second marginals coincide with $\alpha_{1}$ and $\alpha_{2}$. Every measure $\alpha \in \mathcal{M}_{2}(\mathfrak{C})$ on the cone is assigned a measure $\mathfrak{h} \alpha \in \mathcal{M}(X)$ on $X$,

$$
\mathfrak{h} \alpha:=\mathrm{x}_{\#}\left(\mathrm{r}^{2} \alpha\right), \quad(\mathrm{x}, \mathrm{r}): \mathfrak{C} \rightarrow X \times[0,+\infty),(\mathrm{x}, \mathrm{r})([x, r]):=(x, r) \text { for }[x, r] \in \mathfrak{C}, r>0,(\mathrm{x}, \mathrm{r})(\mathfrak{o}):=(\bar{x}, 0),
$$

i.e. $\int_{X} \phi(x) \mathrm{d}(\mathfrak{h} \alpha)=\int_{\mathfrak{C}} \mathrm{r}^{2} \phi(x) \mathrm{d} \alpha$ for all $\phi \in \mathrm{C}_{b}^{0}(X)$. Note that the mapping $\mathfrak{h}: \mathcal{M}_{2}(\mathfrak{C}) \rightarrow \mathcal{M}(X)$ is not injective. It is proved in [23] (see Probl. 7.4, Thm. 7.6, Lem. 7.9, Thm. 7.20 therein) that

$$
\begin{aligned}
\mathrm{HK}_{\Lambda, \Sigma}\left(\mu_{1}, \mu_{2}\right)^{2} & =\min \left\{\mathcal{W}_{\mathfrak{C}, \Lambda, \Sigma}\left(\alpha_{1}, \alpha_{2}\right)^{2} \mid \alpha_{i} \in \mathcal{M}_{2}(\mathfrak{C}), \mathfrak{h} \alpha_{i}=\mu_{i}, i=1,2\right\} \\
& =\min \left\{\mathcal{W}_{\mathfrak{C}, \Lambda, \Sigma}\left(\alpha_{1}, \alpha_{2}\right)^{2}+\frac{4}{\Sigma} \sum_{i=1}^{2}\left(\mu_{i}-\mathfrak{h} \alpha_{i}\right)(X) \mid \alpha_{i} \in \mathcal{M}_{2}(\mathfrak{C}), \mathfrak{h} \alpha_{i} \leq \mu_{i}, i=1,2\right\},
\end{aligned}
$$

and that every optimal plan $\gamma \in \mathcal{M}(X \times X)$ for the Logarithmic Entropy-Transport problem (1.10) gives rise to a solution to (2.7) and vice versa. Moreover, if $\beta \in \mathcal{M}(\mathfrak{C} \times \mathfrak{C})$ is a solution to the transport problem (2.6), (2.4) (which exists by [23], Thm. 7.6) or it is a solution to (2.7), (2.4), then

$$
\beta\left(\left\{\left(\left[x_{1}, r_{1}\right],\left[x_{2}, r_{2}\right]\right) \in \mathfrak{C} \times \mathfrak{C}: r_{1}, r_{2}>0, \mathrm{~d}\left(x_{1}, x_{2}\right)>\pi \sqrt{\Lambda / \Sigma}\right\}\right)=0,
$$


(see [23], Lem. 7.19).

This equivalent characterization of the Hellinger-Kantorovich distance $\mathrm{HK}_{\Lambda, \Sigma}$ has proved extremely useful in [23] for the examination of structural properties; for example, the proofs therein of Proposition 1.3 (1), (2), (3) are based on it. The fact that all the information on transport of mass and creation / annihilation of mass according to (1.10) lies in a pure transportation problem is also a good starting point for our analysis of subdifferentiability properties of $-\mathrm{HK}_{\Lambda, \Sigma}$ in Section 3 . In this context, geodesics in $\left(\mathfrak{C}, \mathrm{d}_{\mathfrak{C}, \Lambda, \Sigma}\right)$ will play a certain role, i.e. curves $\eta:[0,1] \rightarrow \mathfrak{C}$ satisfying $\mathrm{d}_{\mathfrak{C}, \Lambda, \Sigma}(\eta(t), \eta(s))=|t-s| \mathrm{d}_{\mathfrak{C}, \Lambda, \Sigma}(\eta(0), \eta(1))$ for all $s, t \in[0,1]$.

We construct a geodesic which connects $\left[x_{1}, r_{1}\right]$ and $\left[x_{2}, r_{2}\right]$, supposing that $\mathrm{d}\left(x_{1}, x_{2}\right) \leq \pi \sqrt{\Lambda / \Sigma}, r_{1}, r_{2}>0$, and that there exists a geodesic $\mathcal{X}$ in $(X, \mathrm{~d})$ between $x_{1}$ and $x_{2}, x_{1} \neq x_{2}$ (cf. [23], Sect. 8.1): Let us try to find functions $\mathcal{R}:[0,1] \rightarrow[0,+\infty)$ and $\theta:[0,1] \rightarrow[0,1]$ such that $\eta:[0,1] \rightarrow \mathfrak{C}, \eta(t):=[\mathcal{X}(\theta(t)), \mathcal{R}(t)]$, is such geodesic. We note that

$$
\mathrm{d}_{\mathfrak{C}, \Lambda, \Sigma}(\eta(t), \eta(s))^{2}=\frac{4}{\Sigma}\left(\mathcal{R}(t)^{2}+\mathcal{R}(s)^{2}-2 \mathcal{R}(s) \mathcal{R}(t) \cos \left(\sqrt{\Sigma / 4 \Lambda}|\theta(t)-\theta(s)| \mathrm{d}\left(x_{1}, x_{2}\right)\right)\right)=|z(t)-z(s)|_{\mathbb{C}}^{2},
$$

where $z:[0,1] \rightarrow \mathbb{C}$ is the curve in the complex plane $\mathbb{C}$ defined as

$$
z(t):=\frac{2}{\sqrt{\Sigma}} \mathcal{R}(t) \exp \left(i \theta(t) \sqrt{\Sigma / 4 \Lambda} \mathrm{d}\left(x_{1}, x_{2}\right)\right)
$$

and $|\cdot|_{\mathbb{C}}$ denotes the absolute value for complex numbers. If $z$ is a geodesic in the complex plane between $z_{1}:=\frac{2}{\sqrt{\Sigma}} r_{1}$ and $z_{2}:=\frac{2}{\Sigma} r_{2} \exp \left(i \sqrt{\Sigma / 4 \Lambda} \mathrm{d}\left(x_{1}, x_{2}\right)\right)$, then, according to (2.9), the corresponding curve $\eta$ in the cone space, $\eta(t):=[\mathcal{X}(\theta(t)), \mathcal{R}(t)]$, is a geodesic between $\left[x_{1}, r_{1}\right]$ and $\left[x_{2}, r_{2}\right]$. Hence, the condition

$$
z(t)=z_{1}+t\left(z_{2}-z_{1}\right) \quad \text { for all } t \in[0,1]
$$

which means

$$
\begin{aligned}
& \mathcal{R}(t) \cos \left(\theta(t) \sqrt{\Sigma / 4 \Lambda} \mathrm{d}\left(x_{1}, x_{2}\right)\right)=r_{1}+t\left(r_{2} \cos \left(\sqrt{\Sigma / 4 \Lambda} \mathrm{d}\left(x_{1}, x_{2}\right)\right)-r_{1}\right) \quad \text { for all } t \in[0,1], \\
& \mathcal{R}(t) \sin \left(\theta(t) \sqrt{\Sigma / 4 \Lambda} \mathrm{d}\left(x_{1}, x_{2}\right)\right)=t r_{2} \sin \left(\sqrt{\Sigma / 4 \Lambda} \mathrm{d}\left(x_{1}, x_{2}\right)\right) \quad \text { for all } t \in[0,1]
\end{aligned}
$$

yields an appropriate choice for $\mathcal{R}:[0,1] \rightarrow[0,+\infty)$ and $\theta:[0,1] \rightarrow[0,1]$. It is not difficult to see that, by $(2.12)$ and (2.13), $\mathcal{R}$ and $\theta$ are smooth functions, their first derivatives satisfy

$$
\frac{4}{\Sigma}\left(\mathcal{R}^{\prime}(t)\right)^{2}+\frac{1}{\Lambda} \mathcal{R}(t)^{2}\left(\theta^{\prime}(t)\right)^{2} \mathrm{~d}\left(x_{1}, x_{2}\right)^{2}=\mathrm{d}_{\mathfrak{C}, \Lambda, \Sigma}\left(\left[x_{1}, r_{1}\right],\left[x_{2}, r_{2}\right]\right)^{2} \quad \text { for all } t \in(0,1),
$$

and they are right differentiable at $t=0$ with right derivatives

$$
\theta_{+}^{\prime}(0)=\frac{r_{2}}{r_{1}} \frac{\sin \left(\sqrt{\Sigma / 4 \Lambda} \mathrm{d}\left(x_{1}, x_{2}\right)\right)}{\sqrt{\Sigma / 4 \Lambda} \mathrm{d}\left(x_{1}, x_{2}\right)} \quad \text { and } \quad \mathcal{R}_{+}^{\prime}(0)=r_{2} \cos \left(\sqrt{\Sigma / 4 \Lambda} \mathrm{d}\left(x_{1}, x_{2}\right)\right)-r_{1}
$$

Finally, we note that the curves $\eta:[0,1] \rightarrow \mathfrak{C}$,

$$
\eta(t):= \begin{cases}\mathfrak{o} & \text { if } r_{1}+t\left(r_{2}-r_{1}\right)=0 \\ {\left[x, r_{1}+t\left(r_{2}-r_{1}\right)\right]} & \text { else }\end{cases}
$$


are geodesics in $\left(\mathfrak{C}, \mathrm{d}_{\mathfrak{C}, \Lambda, \Sigma}\right)$, connecting $\left[x, r_{1}\right]\left(\right.$ for $\left.r_{1}>0\right)$ or the vertex $\mathfrak{o}\left(\right.$ for $\left.r_{1}=0\right)$ with $\left[x, r_{2}\right]$ (for $r_{2}>0$ ) and with $\mathfrak{o}$ (for $r_{2}=0$ ). They take the above form $\eta(t)=[\mathcal{X}(\theta(t)), \mathcal{R}(t)]$ if we set $\mathcal{X} \equiv x$ (and identify the vertex with $[x, 0]$ if necessary),

$$
\theta \equiv 0 \quad \text { and } \quad \mathcal{R}(t):=r_{1}+t\left(r_{2}-r_{1}\right)
$$

also satisfying (2.14) and the second part of (2.15).

\section{SUPERDIFFERENTIABILITY PROPERTIES OF THE HELLINGER-KANTOROVICH DISTANCE}

Whenever a new distance is introduced, the question of differentiability properties arises. For the class of Hellinger-Kantorovich distances $\mathrm{H}_{\Lambda, \Sigma}, \Lambda, \Sigma>0$, there has not been a corresponding analysis in the literature yet. In this section, we restrict ourselves to studying the superdifferentiability of $\mathrm{HK}_{\Lambda, \Sigma}$ (i.e. subdifferentiability of $-\mathrm{H}_{\Lambda, \Sigma}$ ) along basic directions (1.23) while we postpone studying the differentiability along general absolutely continuous curves to the subsequent paper [11].

Let $\mathbb{H}$ be a separable Hilbert space with scalar product $\langle\cdot, \cdot\rangle$ and norm $\|\cdot\|:=\sqrt{\langle\cdot, \cdot\rangle}$ and let $X \subset \mathbb{H}$ be closed and convex. The couple $(X, \mathrm{~d})$ with $\mathrm{d}\left(x_{1}, x_{2}\right):=\left\|x_{1}-x_{2}\right\|$ forms a Polish space. For $\Lambda, \Sigma>0$, let the space $\mathcal{M}(X)$ of finite nonnegative Radon measures on $X$ be endowed with the distance $\mathrm{HK}_{\Lambda, \Sigma}$.

We fix bounded Borel measurable functions $v: X \rightarrow \mathbb{H}$ and $R: X \rightarrow \mathbb{R}$, supposing that, for $h$ in a neighbourhood $\mathcal{N}$ around 0 , the function $I+h v: X \rightarrow \mathbb{H}$ maps $X$ into $X$, where $I$ denotes the identity mapping $I: \mathbb{H} \rightarrow \mathbb{H}, I(x):=x$, and $1+h R(x)>0$ for all $x \in X$ (which is satisfied whenever $|h|$ is small enough since $R$ is bounded). We define, for a given $\nu_{0} \in \mathcal{M}(X)$, the curve $\mathcal{N} \ni h \mapsto \nu_{h} \in \mathcal{M}(X)$ as

$$
\nu_{h}:=(I+h v)_{\#}(1+h R)^{2} \nu_{0}
$$

i.e. $\int_{X} \phi(x) \mathrm{d} \nu_{h}=\int_{X} \phi(x+h v(x))(1+h R(x))^{2} \mathrm{~d} \nu_{0}$ for all $\phi \in \mathrm{C}_{b}^{0}(X)$. Our goal is to identify elements of the Fréchet subdifferential of

$$
h \mapsto-\frac{1}{2} \mathrm{HK}_{\Lambda, \Sigma}\left(\nu_{h}, \mu\right)^{2} \quad(\mu \in \mathcal{M}(X))
$$

at $h=0$. A good strategy for this is to examine the subdifferentiability issue on the level of the optimal transportation problem on the associated cone $\mathfrak{C}$ first. We refer to Section 2 for notation and details about the optimal transport problem on $\mathfrak{C}$.

Definition 3.1 (Fréchet subdifferential). We say that $\varsigma \in \mathbb{R}$ belongs to the Fréchet subdifferential of a mapping $\mathcal{N} \ni h \mapsto f(h) \in \mathbb{R}$ at $h=0$ if and only if

$$
\liminf _{h \rightarrow 0} \frac{f(h)-f(0)-\varsigma h}{|h|} \geq 0 .
$$

Throughout this section, let $v$ and $R$ be given as above.

Lemma 3.2. For a given $\alpha_{0} \in \mathcal{M}_{2}(\mathfrak{C})$, we define the curve $\mathcal{N} \ni h \mapsto \alpha_{h} \in \mathcal{M}_{2}(\mathfrak{C})$ as

$$
\alpha_{h}:=([x+h v(x), r(1+h R(x))])_{\#} \alpha_{0},
$$

i.e. $\int_{\mathfrak{C}} \varphi([x, r]) \mathrm{d} \alpha_{h}=\int_{\mathfrak{C}} \varphi([x+h v(x), r(1+h R(x))]) \mathrm{d} \alpha_{0}$ for all $\varphi \in \mathrm{C}_{b}^{0}(\mathfrak{C})$. Let $\alpha_{\star} \in \mathcal{M}_{2}(\mathfrak{C})$ be given with $\alpha_{\star}(\mathfrak{C})=\alpha_{0}(\mathfrak{C})$ and let $\beta_{0, \star} \in M\left(\alpha_{0}, \alpha_{\star}\right)$ be optimal in the definition of $\mathcal{W}_{\mathfrak{C}, \Lambda, \Sigma}\left(\alpha_{0}, \alpha_{\star}\right)^{2}$ according to (2.4). We 
suppose that $\beta_{0, \star}$ satisfies (2.8). Then the Fréchet subdifferential of the mapping

$$
h \mapsto-\frac{1}{2} \mathcal{W}_{\mathfrak{C}, \Lambda, \Sigma}\left(\alpha_{h}, \alpha_{\star}\right)^{2}
$$

at $h=0$ is nonempty and

$$
\frac{4}{\Sigma} \int_{\mathfrak{C} \times \mathfrak{C}}\left[-r_{1}^{2} R\left(x_{1}\right)+r_{1} r_{2} R\left(x_{1}\right) \cos \left(\sqrt{\Sigma / 4 \Lambda}\left\|x_{1}-x_{2}\right\|\right)+r_{1} r_{2} \sqrt{\Sigma / 4 \Lambda}\left\langle S_{\Lambda, \Sigma}\left(x_{1}, x_{2}\right), v\left(x_{1}\right)\right\rangle\right] \mathrm{d} \beta_{0, \star}
$$

belongs to it, where

$$
S_{\Lambda, \Sigma}\left(x_{1}, x_{2}\right):= \begin{cases}\frac{\sin \left(\sqrt{\Sigma / 4 \Lambda}|| x_{1}-x_{2} \|\right)}{\left\|x_{1}-x_{2}\right\|}\left(x_{2}-x_{1}\right) & \text { if } x_{1} \neq x_{2}, \\ 0 & \text { if } x_{1}=x_{2} .\end{cases}
$$

Proof. First of all, we note that $\alpha_{h}(\mathfrak{C})=\alpha_{0}(\mathfrak{C})$ and we define

$$
\beta_{h, \star}:=\left(\left[x_{1}+h v\left(x_{1}\right), r_{1}\left(1+h R\left(x_{1}\right)\right)\right],\left[x_{2}, r_{2}\right]\right)_{\#} \beta_{0, \star},
$$

i.e. $\int_{\mathfrak{C} \times \mathfrak{C}} \varphi\left(\left[x_{1}, r_{1}\right],\left[x_{2}, r_{2}\right]\right) \mathrm{d} \beta_{h, \star}=\int_{\mathfrak{C} \times \mathfrak{C}} \varphi\left(\left[x_{1}+h v\left(x_{1}\right), r_{1}\left(1+h R\left(x_{1}\right)\right)\right],\left[x_{2}, r_{2}\right]\right) \mathrm{d} \beta_{0, \star}$ for all $\varphi \in \mathrm{C}_{b}^{0}(\mathfrak{C} \times \mathfrak{C})$. Since $\beta_{h, \star} \in M\left(\alpha_{h}, \alpha_{\star}\right)$, we have

$$
\begin{aligned}
\mathcal{W}_{\mathfrak{C}, \Lambda, \Sigma}\left(\alpha_{h}, \alpha_{\star}\right)^{2} & \leq \int_{\mathfrak{C} \times \mathfrak{C}} \mathrm{d}_{\mathfrak{C}, \Lambda, \Sigma}\left(\left[x_{1}, r_{1}\right],\left[x_{2}, r_{2}\right]\right)^{2} \mathrm{~d} \beta_{h, \star} \\
& =\int_{\mathfrak{C} \times \mathfrak{C}} \mathrm{d}_{\mathfrak{C}, \Lambda, \Sigma}\left(\left[x_{1}+h v\left(x_{1}\right), r_{1}\left(1+h R\left(x_{1}\right)\right)\right],\left[x_{2}, r_{2}\right]\right)^{2} \mathrm{~d} \beta_{0, \star}
\end{aligned}
$$

leading to

$$
\begin{aligned}
& \frac{1}{2}\left(\mathcal{W}_{\mathfrak{C}, \Lambda, \Sigma}\left(\alpha_{0}, \alpha_{\star}\right)^{2}-\mathcal{W}_{\mathfrak{C}, \Lambda, \Sigma}\left(\alpha_{h}, \alpha_{\star}\right)^{2}\right) \\
\geq & \frac{1}{2} \int_{\mathfrak{C} \times \mathfrak{C}}\left[\mathrm{d}_{\mathfrak{C}, \Lambda, \Sigma}\left(\left[x_{1}, r_{1}\right],\left[x_{2}, r_{2}\right]\right)^{2}-\mathrm{d}_{\mathfrak{C}, \Lambda, \Sigma}\left(\left[x_{1}+h v\left(x_{1}\right), r_{1}\left(1+h R\left(x_{1}\right)\right)\right],\left[x_{2}, r_{2}\right]\right)^{2}\right] \mathrm{d} \beta_{0, \star} \\
= & \frac{4}{\Sigma} \int_{\mathfrak{C} \times \mathfrak{C}}\left[-r_{1}^{2} h R\left(x_{1}\right)-\frac{1}{2} h^{2} R\left(x_{1}\right)^{2} r_{1}^{2}+r_{1} r_{2} h R\left(x_{1}\right) \cos \left(\left(\sqrt{\Sigma / 4 \Lambda}\left\|x_{1}+h v\left(x_{1}\right)-x_{2}\right\|\right) \wedge \pi\right)\right] \mathrm{d} \beta_{0, \star} \\
+ & \frac{4}{\Sigma} \int_{\mathfrak{C} \times \mathfrak{C}}\left[r_{1} r_{2} \cos \left(\left(\sqrt{\Sigma / 4 \Lambda}\left\|x_{1}+h v\left(x_{1}\right)-x_{2}\right\|\right) \wedge \pi\right)-r_{1} r_{2} \cos \left(\sqrt{\Sigma / 4 \Lambda}\left\|x_{1}-x_{2}\right\|\right)\right] \mathrm{d} \beta_{0, \star}
\end{aligned}
$$

By dominated convergence theorem, it follows that

$$
\begin{aligned}
& \liminf _{h \downarrow 0} \frac{\frac{1}{2} \mathcal{W}_{\mathfrak{C}, \Lambda, \Sigma}\left(\alpha_{0}, \alpha_{\star}\right)^{2}-\frac{1}{2} \mathcal{W}_{\mathfrak{C}, \Lambda, \Sigma}\left(\alpha_{h}, \alpha_{\star}\right)^{2}}{h} \\
& \geq \frac{4}{\Sigma} \int_{\mathfrak{C} \times \mathfrak{C}}\left[-r_{1}^{2} R\left(x_{1}\right)+r_{1} r_{2} R\left(x_{1}\right) \cos \left(\sqrt{\Sigma / 4 \Lambda}|| x_{1}-x_{2}||\right)+r_{1} r_{2} \sqrt{\Sigma / 4 \Lambda}\left\langle S_{\Lambda, \Sigma}\left(x_{1}, x_{2}\right), v\left(x_{1}\right)\right\rangle\right] \mathrm{d} \beta_{0, \star} \\
& \geq \limsup _{h \uparrow 0} \frac{\frac{1}{2} \mathcal{W}_{\mathfrak{C}, \Lambda, \Sigma}\left(\alpha_{0}, \alpha_{\star}\right)^{2}-\frac{1}{2} \mathcal{W}_{\mathfrak{C}, \Lambda, \Sigma}\left(\alpha_{h}, \alpha_{\star}\right)^{2}}{h}
\end{aligned}
$$

which completes the proof. 
Proposition 3.3. For a given $\nu_{0} \in \mathcal{M}(X)$, we define the curve $\mathcal{N} \ni h \mapsto \nu_{h} \in \mathcal{M}(X)$ as in (3.1). Let $\mu \in \mathcal{M}(X)$ be given and let $\beta_{0, \star} \in \mathcal{M}(\mathfrak{C} \times \mathfrak{C})$ be optimal in the definition of $\mathrm{HK}_{\Lambda, \Sigma}\left(\nu_{0}, \mu\right)^{2}$ according to (2.7), (2.4), with first marginal $\alpha_{0} \in \mathcal{M}_{2}(\mathfrak{C}), \mathfrak{h} \alpha_{0} \leq \nu_{0}$, and second marginal $\alpha_{\star} \in \mathcal{M}_{2}(\mathfrak{C}), \mathfrak{h} \alpha_{\star} \leq \mu$. Then the Fréchet subdifferential of the mapping

$$
h \mapsto-\frac{1}{2} \mathrm{HK}_{\Lambda, \Sigma}\left(\nu_{h}, \mu\right)^{2}
$$

at $h=0$ is nonempty and

$$
\mathfrak{F}_{0, \star, v, R}-\frac{4}{\Sigma} \int_{X} R(x) \mathrm{d}\left(\nu_{0}-\mathfrak{h} \alpha_{0}\right)
$$

belongs to it, where $\mathfrak{F}_{0, \star, v, R}$ is defined as

$$
\frac{4}{\Sigma} \int_{\mathfrak{C} \times \mathfrak{C}}\left[-r_{1}^{2} R\left(x_{1}\right)+r_{1} r_{2} R\left(x_{1}\right) \cos \left(\sqrt{\Sigma / 4 \Lambda}|| x_{1}-x_{2} \|\right)+r_{1} r_{2} \sqrt{\Sigma / 4 \Lambda}\left\langle S_{\Lambda, \Sigma}\left(x_{1}, x_{2}\right), v\left(x_{1}\right)\right\rangle\right] \mathrm{d} \beta_{0, \star},
$$

with $S_{\Lambda, \Sigma}$ as in (3.7).

Proof. We define the curve $\mathcal{N} \ni h \mapsto \alpha_{h} \in \mathcal{M}_{2}(\mathfrak{C})$ as in (3.4). It holds that

$$
\begin{aligned}
\int_{X} \phi(x) \mathrm{d}\left(\mathfrak{h} \alpha_{h}\right) & =\int_{\mathfrak{C}} \mathrm{r}^{2} \phi(\mathrm{x}) \mathrm{d} \alpha_{h}=\int_{\mathfrak{C}} \mathrm{r}^{2}(1+h R(\mathrm{x}))^{2} \phi(\mathrm{x}+h v(\mathrm{x})) \mathrm{d} \alpha_{0}=\int_{X}(1+h R(x))^{2} \phi(x+h v(x)) \mathrm{d}\left(\mathfrak{h} \alpha_{0}\right) \\
& \leq \int_{X}(1+h R(x))^{2} \phi(x+h v(x)) \mathrm{d} \nu_{0}=\int_{X} \phi(x) \mathrm{d} \nu_{h}
\end{aligned}
$$

for all nonnegative bounded Borel functions $\phi: X \rightarrow \mathbb{R}$, see (2.5), from which we infer that

$$
\mathfrak{h} \alpha_{h} \leq \nu_{h} \quad \text { and } \quad\left(\nu_{h}-\mathfrak{h} \alpha_{h}\right)(X)=\int_{X}(1+h R(x))^{2} \mathrm{~d}\left(\nu_{0}-\mathfrak{h} \alpha_{0}\right)
$$

Hence, we have

$$
\begin{aligned}
\frac{1}{2}\left(\mathrm{HK}_{\Lambda, \Sigma}\left(\nu_{0}, \mu\right)^{2}-\mathrm{HK}_{\Lambda, \Sigma}\left(\nu_{h}, \mu\right)^{2}\right) & \geq \frac{1}{2}\left(\mathcal{W}_{\mathfrak{e}, \Lambda, \Sigma}\left(\alpha_{0}, \alpha_{\star}\right)^{2}-\mathcal{W}_{\mathfrak{C}, \Lambda, \Sigma}\left(\alpha_{h}, \alpha_{\star}\right)^{2}\right) \\
& +\frac{2}{\Sigma} \int_{X}\left(-2 h R(x)-h^{2} R(x)^{2}\right) \mathrm{d}\left(\nu_{0}-\mathfrak{h} \alpha_{0}\right),
\end{aligned}
$$

and we conclude by applying Lemma 3.2.

This result can also be expressed in terms of the Logarithmic Entropy-Transport characterization (1.10) of the Hellinger-Kantorovich distance $\mathrm{H}_{\Lambda, \Sigma}$. By Theorem 7.20 in [23], every optimal plan $\gamma \in \mathcal{M}(X \times X)$ for the Logarithmic Entropy-Transport problem (1.10) gives rise to a solution $\beta \in \mathcal{M}(\mathfrak{C} \times \mathfrak{C})$ to the optimal transportation problem $((2.7),(2.4))$ on the cone. Therefore, we obtain

Corollary 3.4. Let $\mu, \nu_{0}, \nu_{h} \in \mathcal{M}(X)$ be given as in Proposition 3.3 and let $\gamma \in \mathcal{M}(X \times X)$ be optimal in the definition of $\mathrm{H}_{\Lambda, \Sigma}\left(\nu_{0}, \mu\right)^{2}$ according to (1.10), with first marginal $\gamma_{0} \ll \nu_{0}$ and second marginal $\gamma_{\star} \ll \mu$. We suppose that

$$
\nu_{0}=\rho_{0} \gamma_{0}+\nu_{0}^{\perp} \quad \text { and } \quad \mu=\rho_{\star} \gamma_{\star}+\mu^{\perp}
$$


for Borel functions $\rho_{0}, \rho_{\star}: X \rightarrow[0,+\infty)$ and nonnegative finite Radon measures $\nu_{0}^{\perp}, \mu^{\perp} \in \mathcal{M}(X), \nu_{0}^{\perp} \perp \gamma_{0}, \mu^{\perp} \perp \gamma_{\star}$, i.e. $\int_{X} \phi(x) \mathrm{d} \nu_{0}=\int_{X} \rho_{0}(x) \phi(x) \mathrm{d} \gamma_{0}+\int_{X} \phi(x) \mathrm{d} \nu_{0}^{\perp}$ for all $\phi \in \mathrm{C}_{b}^{0}(X)$ and there exists a Borel set $B_{0} \subset X$ such that $\nu_{0}^{\perp}\left(B_{0}\right)=0=\gamma_{0}\left(X \backslash B_{0}\right)$; similarly for $\left(\mu, \rho_{\star}, \gamma_{\star}, \mu^{\perp}\right)$. Then

$$
\mathfrak{F}_{0, \star, v, R}-\frac{4}{\Sigma} \int_{X} R(x) \mathrm{d} \nu_{0}^{\perp}
$$

belongs to the Fréchet subdifferential of (3.8) at $h=0$, with $\mathfrak{F}_{0, \star, v, R}$ defined as

$$
\begin{aligned}
& \frac{4}{\Sigma} \int_{X \times X}\left[-\rho_{0}\left(x_{1}\right) R\left(x_{1}\right)+\sqrt{\rho_{0}\left(x_{1}\right) \rho_{\star}\left(x_{2}\right)} R\left(x_{1}\right) \cos \left(\sqrt{\Sigma / 4 \Lambda}\left\|x_{1}-x_{2}\right\|\right)\right] \mathrm{d} \gamma \\
+ & \frac{4}{\Sigma} \int_{X \times X} \sqrt{\Sigma / 4 \Lambda \rho_{0}\left(x_{1}\right) \rho_{\star}\left(x_{2}\right)}\left\langle S_{\Lambda, \Sigma}\left(x_{1}, x_{2}\right), v\left(x_{1}\right)\right\rangle \mathrm{d} \gamma .
\end{aligned}
$$

Proof. We define

$$
\beta_{0, \star}:=\left(\left[x_{1}, \sqrt{\rho_{0}\left(x_{1}\right)}\right],\left[x_{2}, \sqrt{\rho_{\star}\left(x_{2}\right)}\right]\right)_{\#} \gamma \in \mathcal{M}(\mathfrak{C} \times \mathfrak{C})
$$

i.e. $\int_{\mathfrak{C} \times \mathfrak{C}} \varphi\left(\left[x_{1}, r_{1}\right],\left[x_{2}, r_{2}\right]\right) \mathrm{d} \beta_{0, \star}=\int_{X \times X} \varphi\left(\left[x_{1}, \sqrt{\rho_{0}\left(x_{1}\right)}\right],\left[x_{2}, \sqrt{\rho_{\star}\left(x_{2}\right)}\right]\right) \mathrm{d} \gamma$ for all $\varphi \in \mathrm{C}_{b}^{0}(\mathfrak{C} \times \mathfrak{C})$. According to Theorem 7.20 (iii) in [23], $\beta_{0, \star}$ is a solution to $((2.7),(2.4))$. By Proposition 3.3, the claim is proved.

We remark that, according to Theorem 1.115 in [13] or Lemma 2.3 in [23], such 'Lebesgue decomposition' (3.11) always exists.

Our analysis of the Fréchet subdifferentials of the mappings (3.8) at $h=0$ will form the basis for the general study of differentiability properties of the Hellinger-Kantorovich distance $\mathrm{K}_{\Lambda, \Sigma}$ in [11]. For the purposes of our Minimizing Movement approach to (1.21), (1.22), the results from Proposition 3.3 and Corollary 3.4 will be sufficient. We conclude this section with a link between the Fréchet subdifferential of (3.8) at $h=0$ for $v:=\frac{4 \Lambda}{\Sigma} \nabla \phi, R:=2 \phi$, and the difference

$$
\int_{X} \phi(x) \mathrm{d} \mu-\int_{X} \phi(x) \mathrm{d} \nu_{0}
$$

Proposition 3.5. Let $\phi: \mathbb{H} \rightarrow \mathbb{R}$ be a twice continuously differentiable function whose differentials of first and second order at $x \in \mathbb{H}$ are represented by the gradient $\nabla \phi(x) \in \mathbb{H}$ and the Hessian $\nabla^{2} \phi(x): \mathbb{H} \rightarrow \mathbb{H}$ respectively. We suppose that

$$
C_{\phi}:=\sup _{x \in X}\left(|\phi(x)|+\|\nabla \phi(x)\|+||\left|\nabla^{2} \phi(x) \|\right| \mid\right)<+\infty
$$

with

$$
\left\|\nabla^{2} \phi(x)\right\| \|:=\sup \left\{\left\|\nabla^{2} \phi(x)(v)\right\|: \quad v \in \mathbb{H},\|v\| \leq 1\right\}
$$

For $\nu_{0}, \mu \in \mathcal{M}(X)$, let $\beta_{0, \star} \in \mathcal{M}(\mathfrak{C} \times \mathfrak{C})$ be optimal in the definition of $\mathrm{H}_{\Lambda, \Sigma}\left(\nu_{0}, \mu\right)^{2}$ according to (2.7), (2.4), with first marginal $\alpha_{0} \in \mathcal{M}_{2}(\mathfrak{C}), \mathfrak{h} \alpha_{0} \leq \nu_{0}$, and second marginal $\alpha_{\star} \in \mathcal{M}_{2}(\mathfrak{C}), \mathfrak{h} \alpha_{\star} \leq \mu$. Then the following holds good:

$$
\left|\frac{4}{\Sigma}\left(\int_{X} \phi(x) \mathrm{d} \mu-\int_{X} \phi(x) \mathrm{d} \nu_{0}\right)-\left(\mathfrak{F}_{0, \star, \phi}-\frac{8}{\Sigma} \int_{X} \phi(x) \mathrm{d}\left(\nu_{0}-\mathfrak{h} \alpha_{0}\right)\right)\right| \leq C_{\phi}(6+16 \Lambda / \Sigma) \mathrm{H} \mathrm{K}_{\Lambda, \Sigma}\left(\nu_{0}, \mu\right)^{2},
$$


where $\mathfrak{F}_{0, \star, \phi}$ is defined as

$$
\frac{4}{\Sigma} \int_{\mathfrak{C} \times \mathfrak{C}}\left[-2 r_{1}^{2} \phi\left(x_{1}\right)+2 r_{1} r_{2} \phi\left(x_{1}\right) \cos \left(\sqrt{\Sigma / 4 \Lambda}\left\|x_{1}-x_{2}\right\|\right)+r_{1} r_{2} \sqrt{4 \Lambda / \Sigma}\left\langle S_{\Lambda, \Sigma}\left(x_{1}, x_{2}\right), \nabla \phi\left(x_{1}\right)\right\rangle\right] \mathrm{d} \beta_{0, \star}
$$

(with $S_{\Lambda, \Sigma}$ as in (3.7)).

Proof. First of all, we note that

$$
\int_{X} \phi(x) \mathrm{d} \mu-\int_{X} \phi(x) \mathrm{d} \nu_{0}=\int_{\mathfrak{C} \times \mathfrak{C}}\left[\phi\left(x_{2}\right) r_{2}^{2}-\phi\left(x_{1}\right) r_{1}^{2}\right] \mathrm{d} \beta_{0, \star}+\int_{X} \phi(x) \mathrm{d}\left(\mu-\mathfrak{h} \alpha_{\star}\right)-\int_{X} \phi(x) \mathrm{d}\left(\nu_{0}-\mathfrak{h} \alpha_{0}\right)
$$

and

$$
\left|\frac{4}{\Sigma} \int_{X} \phi(x) \mathrm{d}\left(\mu-\mathfrak{h} \alpha_{\star}\right)-\frac{4}{\Sigma} \int_{X} \phi(x) \mathrm{d}\left(\nu_{0}-\mathfrak{h} \alpha_{0}\right)+\frac{8}{\Sigma} \int_{X} \phi(x) \mathrm{d}\left(\nu_{0}-\mathfrak{h} \alpha_{0}\right)\right| \leq C_{\phi} \mathfrak{H K}_{\Lambda, \Sigma}\left(\nu_{0}, \mu\right)^{2}
$$

Hence, all that remains is to find a suitable estimate of $\left|\frac{4}{\Sigma} \int_{\mathfrak{C} \times \mathfrak{C}}\left[\phi\left(x_{2}\right) r_{2}^{2}-\phi\left(x_{1}\right) r_{1}^{2}\right] \mathrm{d} \beta_{0, \star}-\mathfrak{F}_{0, \star, \phi}\right|$. We fix $\left(\left[x_{1}, r_{1}\right],\left[x_{2}, r_{2}\right]\right) \in \mathfrak{C} \times \mathfrak{C} \backslash\{(\mathfrak{o}, \mathfrak{o})\}$ with $\left\|x_{1}-x_{2}\right\| \leq \pi \sqrt{\Lambda / \Sigma}$. Let $\eta:[0,1] \rightarrow \mathfrak{C}, \eta(t):=\left[x_{1}+\theta(t)\left(x_{2}-\right.\right.$ $\left.\left.x_{1}\right), \mathcal{R}(t)\right]$, be the geodesic between $\left[x_{1}, r_{1}\right]$ and $\left[x_{2}, r_{2}\right]$ in $\left(\mathfrak{C}, \mathrm{d}_{\mathfrak{C}, \Lambda, \Sigma}\right)$, defined according to (2.9)-(2.17). Then the mapping $t \mapsto \chi(t):=\phi\left(x_{1}+\theta(t)\left(x_{2}-x_{1}\right)\right) \mathcal{R}(t)^{2}$ is twice continuously differentiable with

$$
\begin{aligned}
\chi^{\prime}(t)= & 2 \mathcal{R}(t) \mathcal{R}^{\prime}(t) \phi\left(x_{1}+\theta(t)\left(x_{2}-x_{1}\right)\right)+\mathcal{R}(t)^{2} \theta^{\prime}(t)\left\langle\nabla \phi\left(x_{1}+\theta(t)\left(x_{2}-x_{1}\right)\right), x_{2}-x_{1}\right\rangle \\
\chi^{\prime \prime}(t)= & \left(\frac{\mathrm{d}}{\mathrm{d}^{2} t} \mathcal{R}(t)^{2}\right) \phi\left(x_{1}+\theta(t)\left(x_{2}-x_{1}\right)\right)+2\left(\frac{\mathrm{d}}{\mathrm{d} t} \mathcal{R}(t)^{2}\right) \theta^{\prime}(t)\left\langle\nabla \phi\left(x_{1}+\theta(t)\left(x_{2}-x_{1}\right)\right), x_{2}-x_{1}\right\rangle \\
& +\mathcal{R}(t)^{2} \theta^{\prime \prime}(t)\left\langle\nabla \phi\left(x_{1}+\theta(t)\left(x_{2}-x_{1}\right)\right), x_{2}-x_{1}\right\rangle \\
& +\mathcal{R}(t)^{2}\left(\theta^{\prime}(t)\right)^{2}\left\langle x_{2}-x_{1}, \nabla^{2} \phi\left(x_{1}+\theta(t)\left(x_{2}-x_{1}\right)\right)\left(x_{2}-x_{1}\right)\right\rangle
\end{aligned}
$$

for $t \in(0,1)$ and it is right differentiable at $t=0$ with right derivative

$$
\begin{aligned}
\chi_{+}^{\prime}(0)=\lim _{t \downarrow 0} \chi^{\prime}(t) & =2 r_{1} \mathcal{R}_{+}^{\prime}(0) \phi\left(x_{1}\right)+r_{1}^{2} \theta_{+}^{\prime}(0)\left\langle\nabla \phi\left(x_{1}\right), x_{2}-x_{1}\right\rangle \\
& =-2 r_{1}^{2} \phi\left(x_{1}\right)+2 r_{1} r_{2} \phi\left(x_{1}\right) \cos \left(\sqrt{\Sigma / 4 \Lambda}\left\|x_{1}-x_{2}\right\|\right)+r_{1} r_{2} \sqrt{4 \Lambda / \Sigma}\left\langle S_{\Lambda, \Sigma}\left(x_{1}, x_{2}\right), \nabla \phi\left(x_{1}\right)\right\rangle .
\end{aligned}
$$

Approximating $\chi$ by the first Taylor polynomial at $t=0$ yields

$$
\left|\phi\left(x_{2}\right) r_{2}^{2}-\phi\left(x_{1}\right) r_{1}^{2}-\chi_{+}^{\prime}(0)\right|=\left|\chi(1)-\chi(0)-\chi_{+}^{\prime}(0)\right| \leq \sup _{t \in(0,1)}\left|\chi^{\prime \prime}(t)\right| .
$$

So let us fix $t \in(0,1)$ and estimate $\left|\chi^{\prime \prime}(t)\right|$. For this, we need to play with the first and second derivatives of $\mathcal{R}$ and $\theta$. We recall $(2.14)$, which says

$$
\frac{4}{\Sigma}\left(\mathcal{R}^{\prime}(t)\right)^{2}+\frac{1}{\Lambda} \mathcal{R}(t)^{2}\left(\theta^{\prime}(t)\right)^{2}\left\|x_{1}-x_{2}\right\|^{2}=\mathrm{d}_{\mathfrak{C}, \Lambda, \Sigma}\left(\left[x_{1}, r_{1}\right],\left[x_{2}, r_{2}\right]\right)^{2} .
$$

It is not difficult to see that (2.12) and (2.13) imply

$$
\frac{4}{\Sigma}\left(\mathcal{R}^{\prime}(t)\right)^{2}+\frac{4}{\Sigma} \mathcal{R}(t) \mathcal{R}^{\prime \prime}(t)=\frac{2}{\Sigma} \frac{\mathrm{d}}{\mathrm{d}^{2} t} \mathcal{R}(t)^{2}=\mathrm{d}_{\mathfrak{C}, \Lambda, \Sigma}\left(\left[x_{1}, r_{1}\right],\left[x_{2}, r_{2}\right]\right)^{2}
$$


We infer from (2.14) and (3.15) that

$$
\frac{1}{\Lambda} \mathcal{R}(t)\left(\theta^{\prime}(t)\right)^{2}\left\|x_{1}-x_{2}\right\|^{2}=\frac{4}{\Sigma} \mathcal{R}^{\prime \prime}(t)
$$

(since $\mathcal{R}(t) \neq 0$ ) and, by taking the derivative in (3.15), that

$$
3 \mathcal{R}^{\prime}(t) \mathcal{R}^{\prime \prime}(t)+\mathcal{R}(t) \mathcal{R}^{\prime \prime \prime}(t)=0
$$

It follows that

$$
\begin{aligned}
\frac{1}{\Lambda} \mathcal{R}(t) \mathcal{R}^{\prime}(t)\left(\theta^{\prime}(t)\right)^{2}\left\|x_{1}-x_{2}\right\|^{2} & =\frac{4}{\Sigma} \mathcal{R}^{\prime}(t) \mathcal{R}^{\prime \prime}(t)=-\frac{4}{3 \Sigma} \mathcal{R}(t) \mathcal{R}^{\prime \prime \prime}(t) \\
& =-\frac{1}{3 \Lambda} \mathcal{R}(t) \mathcal{R}^{\prime}(t)\left(\theta^{\prime}(t)\right)^{2}\left\|x_{1}-x_{2}\right\|^{2}-\frac{2}{3 \Lambda} \mathcal{R}(t)^{2} \theta^{\prime}(t) \theta^{\prime \prime}(t)\left\|x_{1}-x_{2}\right\|^{2}
\end{aligned}
$$

Supposing that $x_{1} \neq x_{2}$ and that $\theta^{\prime}(t) \neq 0$, we obtain

$$
\left|\theta^{\prime \prime}(t) \mathcal{R}(t)^{2}\left\|x_{1}-x_{2}\right\|\right| \leq 2\left|\mathcal{R}^{\prime}(t) \mathcal{R}(t) \theta^{\prime}(t)\left\|x_{1}-x_{2}\right\|\right| \leq\left(\mathcal{R}^{\prime}(t)\right)^{2}+\mathcal{R}(t)^{2}\left(\theta^{\prime}(t)\right)^{2}\left\|x_{1}-x_{2}\right\|^{2} .
$$

We note that (3.16) also holds good if $x_{1}=x_{2}$ or if $\theta^{\prime}(t)=0$, since, taking the first and second derivative in (2.14), we see that $\theta^{\prime}(t)=0$ implies $\theta^{\prime \prime}(t)=0$ or $x_{1}=x_{2}$. Indeed, if $\theta^{\prime}(t)=0$, we have

$$
\mathcal{R}^{\prime \prime}(t)=0=\mathcal{R}^{\prime \prime \prime}(t)
$$

by the above considerations, and thus,

$$
0=\frac{\mathrm{d}}{\mathrm{d}^{2} t} \mathrm{~d}_{\mathfrak{C}, \Lambda, \Sigma}\left(\left[x_{1}, r_{1}\right],\left[x_{2}, r_{2}\right]\right)^{2}=\frac{2}{\Lambda} \mathcal{R}(t)^{2}\left(\theta^{\prime \prime}(t)\right)^{2}\left\|x_{1}-x_{2}\right\|^{2} .
$$

Finally, we obtain

$$
\begin{aligned}
\left|\chi^{\prime \prime}(t)\right| & \leq C_{\phi}(\frac{\mathrm{d}}{\mathrm{d}^{2} t} \mathcal{R}(t)^{2}+\underbrace{2\left|\left(\frac{\mathrm{d}}{\mathrm{d} t} \mathcal{R}(t)^{2}\right) \theta^{\prime}(t)\left\|x_{1}-x_{2}\right\|\right|}_{\leq 2\left(\mathcal{R}^{\prime}(t)\right)^{2}+2 \mathcal{R}(t)^{2}\left(\theta^{\prime}(t)\right)^{2}\left\|x_{1}-x_{2}\right\|^{2}}+\left|\mathcal{R}(t)^{2} \theta^{\prime \prime}(t)\left\|x_{1}-x_{2}\right\|\right|+\mathcal{R}(t)^{2}\left(\theta^{\prime}(t)\right)^{2}\left\|x_{1}-x_{2}\right\|^{2}) \\
& \leq C_{\phi}(5 / 4 \Sigma+4 \Lambda) \mathrm{d}_{\mathfrak{C}, \Lambda, \Sigma}\left(\left[x_{1}, r_{1}\right],\left[x_{2}, r_{2}\right]\right)^{2},
\end{aligned}
$$

by applying (2.14), (3.15) and (3.16), and the fact that $\frac{\mathrm{d}}{\mathrm{d} t} \mathcal{R}(t)^{2}=2 \mathcal{R}^{\prime}(t) \mathcal{R}(t)$.

Note that $\beta_{0, \star}$ satisfies (2.8). All in all, it follows that

$$
\begin{aligned}
\left|\frac{4}{\Sigma} \int_{\mathfrak{C} \times \mathfrak{C}}\left[\phi\left(x_{2}\right) r_{2}^{2}-\phi\left(x_{1}\right) r_{1}^{2}\right] \mathrm{d} \beta_{0, \star}-\mathfrak{F}_{0, \star, \phi}\right| & \leq C_{\phi}(5+16 \Lambda / \Sigma) \int_{\mathfrak{C} \times \mathfrak{C}} \mathrm{d}_{\mathfrak{C}, \Lambda, \Sigma}\left(\left[x_{1}, r_{1}\right],\left[x_{2}, r_{2}\right]\right)^{2} \mathrm{~d} \beta_{0, \star} \\
& \leq C_{\phi}(5+16 \Lambda / \Sigma) \mathrm{H}_{\Lambda, \Sigma}\left(\nu_{0}, \mu\right)^{2} .
\end{aligned}
$$

The proof of Proposition 3.5 is complete.

Remark 3.6. Let $\phi: \mathbb{H} \rightarrow \mathbb{R}$ satisfy the assumptions of Proposition 3.5. In addition, we suppose that $\phi$ has compact support within the interior of $X$ so that, setting $v:=\frac{4 \Lambda}{\Sigma} \nabla \phi$ and $R:=2 \phi$, we can define the curve 
$\mathcal{N} \ni h \mapsto \nu_{h} \in \mathcal{M}(X)$ according to (3.1). Then

$$
\mathfrak{F}_{0, \star, \phi}-\frac{8}{\Sigma} \int_{X} \phi(x) \mathrm{d}\left(\nu_{0}-\mathfrak{h} \alpha_{0}\right),
$$

defined as in Proposition 3.5, belongs to the Fréchet subdifferential of

$$
h \mapsto-\frac{1}{2} \mathrm{H}_{\Lambda, \Sigma}\left(\nu_{h}, \mu\right)^{2}
$$

at $h=0$, see Proposition 3.3.

\section{Minimizing MOVEMENT APPROACH}

\subsection{Theorem and Assumptions}

Let us return to the setting described in Section 1.2 with $X:=\bar{\Omega} \subset \mathbb{R}^{d}, \mathrm{~d}\left(x_{1}, x_{2}\right):=\left|x_{1}-x_{2}\right|$, and let us define $\Phi(\tau, \mu, \nu):=\mathcal{E}(\nu)+\frac{1}{2 \tau} \mathrm{K}_{\Lambda, \Sigma}(\nu, \mu)^{2}, \mathcal{E}:=\mathcal{F}+\mathcal{V}$, as in (1.19) and (1.20). Our goal is to prove that the associated Minimizing Movement scheme yields weak solutions to the scalar reaction-diffusion equation (1.21) with no-flux boundary condition (1.22). In order to find appropriate assumptions on $F:[0,+\infty) \rightarrow \mathbb{R}$ and $V: \bar{\Omega} \rightarrow \mathbb{R}$, it is worth taking a look at the natural coercivity assumptions which typically arise in connection with the Minimizing Movement approach to gradient flows (cf. the fundamental book [2] by Ambrosio, Gigli and Savaré). Note that, by Proposition 1.3, $\left(\mathcal{M}(\bar{\Omega}), \mathrm{HK}_{\Lambda, \Sigma}\right)$ is a complete metric space.

Proposition 4.1 (Minimizing Movement approach to gradient flows [2]). Let $(\mathscr{S}, d)$ be a complete metric space and apply the Minimizing Movement scheme $(1.1)$ to $\Phi(\tau, v, x):=\mathcal{E}(x)+\frac{1}{2 \tau} d(x, v)^{2}$. We suppose that the functional $\mathcal{E}: \mathscr{S} \rightarrow(-\infty,+\infty]$ satisfies the following assumptions:

(A1) There exist $A, B>0, x_{\star} \in \mathscr{S}$ such that

$$
\mathcal{E}(\cdot) \geq-A-B d\left(\cdot, x_{\star}\right)^{2} .
$$

(A2) $\mathcal{E}$ is lower semicontinuous, i.e.

$$
d\left(x_{n}, x\right) \rightarrow 0 \Rightarrow \liminf _{n \rightarrow \infty} \mathcal{E}\left(x_{n}\right) \geq \mathcal{E}(x) .
$$

(A3) Every d-bounded set contained in a sublevel of $\mathcal{E}$ is relatively compact, i.e.

$$
\sup _{n, m}\left\{\mathcal{E}\left(x_{n}\right), d\left(x_{n}, x_{m}\right)\right\}<+\infty \quad \Rightarrow \quad \exists n_{k} \uparrow+\infty, x \in \mathscr{S}: d\left(x_{n_{k}}, x\right) \rightarrow 0 .
$$

Then, for every $u_{0} \in\{\mathcal{E}<+\infty\}$, the set of Generalized Minimizing Movements $\operatorname{GMM}\left(\Phi ; u_{0}\right)$ is nonempty. Moreover, every $u \in \operatorname{GMM}\left(\Phi ; u_{0}\right)$ is continuous (locally absolutely continuous even) and satisfies the energy dissipation inequality (1.5).

Proof. See Chapters 1-3 in [2]. The definitions asscociated with the energy dissipation inequality (1.5) can be found therein, too. A brief outline of (1.5) is given in Section 4.3 in this paper.

So let us break down the Assumptions (A1), (A2) and (A3) of Proposition 4.1 on $\mathcal{E}$ into assumptions on $F$ and $V$. Let us put the focus on $F$ first, supposing that $V \equiv 0$. We start with Ass. (A1). We notice that for a Borel measurable function $F:[0,+\infty) \rightarrow \mathbb{R}$, we have

$$
\int_{\Omega} \min \{F(u(x)), 0\} \mathrm{d} x>-\infty
$$


for all $u: \Omega \rightarrow[0,+\infty), u \in \mathrm{L}^{1}(\Omega)$, if and only if $F$ is linearly bounded from below, i.e. condition (4.4) below is a necessary and sufficient condition for the well-posedness of $\mathcal{F}$ ( $c f$. Theorem 5.1 and Ex. 5.5 in [13]).

Lemma 4.2. We suppose that $F:[0,+\infty) \rightarrow \mathbb{R}$ is Borel measurable and there exists $C_{F}>0$ such that

$$
F(s) \geq-C_{F} s-C_{F} \quad \text { for all } s \in[0,+\infty) .
$$

Let $\mathcal{F}$ be defined as in (1.20) and let $\eta_{0}$ denote the null measure. Then there exist $A, B>0$ such that

$$
\mathcal{F}(\cdot) \geq-A-B \mathrm{HK}_{\Lambda, \Sigma}\left(\cdot, \eta_{0}\right)^{2} .
$$

Proof. Obviously, (4.4) implies (4.5) with $A:=C_{F} \mathscr{L}^{d}(\Omega)$ and $B:=\frac{\Sigma}{4} C_{F}$; the only thing to note is that we have $\frac{\Sigma}{4} \mathrm{H}_{\Lambda, \Sigma}\left(\mu, \eta_{0}\right)^{2}=\int_{\Omega} u(x) \mathrm{d} x$ if $\mu=u \mathscr{L}^{d}$ by Proposition 1.3 and $\mathscr{L}^{d}(\Omega)<+\infty$ since $\Omega$ is bounded.

As $\mathrm{HK}_{\Lambda, \Sigma}$ metrizes the weak topology on $\mathcal{M}(\bar{\Omega})$ in duality with continuous and bounded functions, according to Proposition 1.3, compactness issues such as (4.3) are closely linked to an application of Prokhorov's Theorem. Thus the compactness of $(\bar{\Omega},|\cdot|)$ yields the relative compactness of every $\mathrm{HK}_{\Lambda, \Sigma}$-bounded set in $\left(\mathcal{M}(\bar{\Omega}), \mathrm{HK}_{\Lambda, \Sigma}\right)$ (cf. [23], Thm. 2.2, Cor. 7.16), i.e. (4.3) holds good in any case. However, this does not suffice in view of Assumption (A2) of Proposition 4.1 and the fact that we aim to obtain (weak) solutions $u:[0,+\infty) \times \Omega \rightarrow$ $[0,+\infty)$ to $(1.21),(1.22)$. We need a condition on $F$ which guarantees

$$
\sup _{n}\left\{\mathcal{F}\left(\mu_{n}\right), \mathrm{K}_{\Lambda, \Sigma}\left(\mu_{n}, \eta_{0}\right)\right\}<+\infty \quad \Rightarrow \quad \exists n_{k} \uparrow+\infty, u: \Omega \rightarrow[0,+\infty): \mathrm{HK}_{\Lambda, \Sigma}\left(\mu_{n_{k}}, u \mathscr{L}^{d}\right) \rightarrow 0
$$

Lemma 4.3. We suppose that $F$ is Borel measurable and linearly bounded from below (4.4). Let $\mathcal{F}$ be defined as in (1.20). Then (4.6) holds good if and only if $F$ has superlinear growth, i.e.

$$
\lim _{s \rightarrow \infty} \frac{F(s)}{s}=+\infty
$$

and it is equivalent to

$$
\sup _{n}\left\{\mathcal{F}\left(\mu_{n}\right), \mathrm{K}_{\Lambda, \Sigma}\left(\mu_{n}, \eta_{0}\right)\right\}<+\infty, \mu_{n}=u_{n} \mathscr{L}^{d} \Rightarrow \exists n_{k} \uparrow+\infty, u: \Omega \rightarrow[0,+\infty): u_{n_{k}} \stackrel{\mathrm{L}^{1}}{\rightarrow} u .
$$

Furthermore, $\mathcal{F}$ is lower semicontinuous, i.e.

$$
\mathrm{H}_{\Lambda, \Sigma}\left(\mu_{n}, \mu\right) \rightarrow 0 \Rightarrow \liminf _{n \rightarrow \infty} \mathcal{F}\left(\mu_{n}\right) \geq \mathcal{F}(\mu)
$$

if and only if $F$ is convex, lower semicontinuous and has superlinear growth (4.7).

Proof. We suppose that $F$ has superlinear growth, $\sup _{n} \mathcal{F}\left(\mu_{n}\right)<+\infty, \mu_{n}=u_{n} \mathscr{L}^{d}$ and $\sup _{n} \int_{\Omega} u_{n}(x) \mathrm{d} x=$ $\frac{\Sigma}{4} \sup _{n} \mathrm{H}_{\Lambda, \Sigma}\left(\mu_{n}, \eta_{0}\right)^{2}<+\infty$. By (4.7), for every $M \in \mathbb{N}$ there exists $s_{M}>0$ such that $F(s) \geq M s$ for all $s \geq s_{M}$. Let a Borel set $E \subset \Omega$ be given. We fix $n \in \mathbb{N}$ and define $E_{M}:=E \cap\left\{x \in \Omega: u_{n}(x)>s_{M}\right\},(M \in \mathbb{N})$. We have

$$
\begin{aligned}
\int_{E} u_{n}(x) \mathrm{d} x & \leq s_{M} \mathscr{L}^{d}(E)+\frac{1}{M} \int_{E_{M}} F\left(u_{n}(x)\right) \mathrm{d} x \\
& \leq s_{M} \mathscr{L}^{d}(E)+\frac{1}{M}\left(\mathcal{F}\left(u_{n}\right)+C_{F} \int_{\Omega} u_{n}(x) \mathrm{d} x+C_{F} \mathscr{L}^{d}(\Omega)\right),
\end{aligned}
$$


which shows the equiintegrability of $\left(u_{n}\right)_{n}$. Therefore, by Dunford-Pettis-Theorem, there exist a subsequence $n_{k} \uparrow+\infty$ and $u: \Omega \rightarrow[0,+\infty)$ such that $u_{n_{k}} \stackrel{\mathrm{L}^{1}}{\rightarrow} u$, where $\stackrel{\mathrm{L}^{1}}{\rightarrow}$ denotes weak convergence in $\mathrm{L}^{1}(\Omega)$. Clearly, $u_{n_{k}} \stackrel{L^{1}}{\rightarrow} u$ implies $\mathrm{H}_{\Lambda, \Sigma}\left(\mu_{n_{k}}, u \mathscr{L}^{d}\right) \rightarrow 0$.

Now, let us suppose that $F$ does not have superlinear growth (4.7). Then there exists $s_{n} \rightarrow \infty$ such that $\sup _{n} \frac{F\left(s_{n}\right)}{s_{n}}<+\infty$. We fix $\bar{x} \in \Omega$. Let $B_{n}$ be the open ball around $\bar{x}$ with radius $\left(\frac{1}{s_{n}}\right)^{1 / d}$, and let $\mu_{n}:=$ $u_{n} \mathscr{L}^{d}, u_{n}(x):=s_{n}$ if $x \in \Omega \cap B_{n}, u_{n}(x):=0$ else. Then $\sup _{n}\left\{\mathcal{F}\left(\mu_{n}\right), \mathrm{HK}_{\Lambda, \Sigma}\left(\mu_{n}, \eta_{0}\right)\right\}<+\infty$ and $\mu_{n}$ converges to the Dirac measure $\operatorname{Vol}_{d} \delta_{\bar{x}}$ (where $\mathrm{Vol}_{d}$ denotes the volume of the unit ball in $\mathbb{R}^{d}$ ) in $\left(\mathcal{M}(\bar{\Omega}), \mathrm{H}_{\Lambda, \Sigma}\right)$. Hence, (4.6) does not hold.

So we have proved that (4.6) holds good if and only if $F$ has superlinear growth and it is equivalent to (4.8).

The second part of the lemma follows from the first part, (4.4) and the fact that, for a Borel measurable function $G: \mathbb{R} \rightarrow[0,+\infty)$, the functional $\mathcal{G}: \mathrm{L}^{1}(\Omega) \rightarrow[0,+\infty], \mathcal{G}(u):=\int_{\Omega} G(u(x)) \mathrm{d} x$, is lower semicontinuous w.r.t. weak $\mathrm{L}^{1}$-convergence if and only if $G$ is convex and lower semicontinuous (cf. Thms. 5.9 and 5.14 in [13]).

Note that, if $F$ is convex, then it is automatically linearly bounded from below, i.e. there exists $C_{F}>0$ such that (4.4) holds good. We have seen so far that $\mathcal{F}$ satisfies the assumptions of Proposition 4.1 if and only if $F$ is convex, lower semicontinuous and has superlinear growth (4.7). In addition, the proof of (a weak form of) the reaction-diffusion equation (1.21) with no-flux boundary condition (1.22) will require a sort of differentiability property of $\mathcal{F}$, see Assumption 4.7 below. This condition will arise quite naturally.

Now, our theorem reads as follows.

Theorem 4.4. Let a continuous strictly convex function $F:[0,+\infty) \rightarrow \mathbb{R}$ with superlinear growth (4.7) and a Lipschitz continuous function $V: \bar{\Omega} \rightarrow \mathbb{R}$ be given and define $\mathcal{E}:=\mathcal{F}+\mathcal{V}: \mathcal{M}(\bar{\Omega}) \rightarrow(-\infty,+\infty]$ and $\Phi$ according to (1.20) and (1.19). Let $F$ be differentiable in $(0,+\infty)$ and define $L_{F}, \hat{L}_{F}:[0,+\infty) \rightarrow \mathbb{R}$ as

$$
L_{F}(s):=\left\{\begin{array}{ll}
s F^{\prime}(s)-F(s) & \text { if } s \in(0,+\infty), \\
-F(0) & \text { if } s=0,
\end{array} \quad \hat{L}_{F}(s):=L_{F}(s)+F(s)= \begin{cases}s F^{\prime}(s) & \text { if } s \in(0,+\infty), \\
0 & \text { if } s=0 .\end{cases}\right.
$$

We suppose that $\mathcal{F}$ satisfies Assumption 4.7 (see below).

Then the following holds good: For every $\mu_{0} \in\{\mathcal{E}<+\infty\}$, the set $\operatorname{GMM}\left(\Phi ; \mu_{0}\right)$ is nonempty. Furthermore, if $\mu \in \operatorname{GMM}\left(\Phi ; \mu_{0}\right)$, then there exists a curve $u:[0,+\infty) \rightarrow \mathrm{L}^{1}(\Omega), u \geq 0$, such that

$$
\begin{aligned}
& \mu(t)=u(t) \mathscr{L}^{d}, \\
& u\left(t_{n}\right) \stackrel{\mathrm{L}^{1}}{\rightarrow} u(t) \quad \text { if } t_{n} \rightarrow t,
\end{aligned}
$$

for all $t \geq 0$, and $u$ is a solution to a weak version of the reaction-diffusion equation (1.21) with no-flux boundary condition (1.22), i.e.

$$
\hat{L}_{F}(u) \in \mathrm{L}_{\mathrm{loc}}^{2}\left([0,+\infty) ; \mathrm{L}^{1}(\Omega)\right), \quad L_{F}(u) \in \mathrm{L}_{\mathrm{loc}}^{2}\left([0,+\infty) ; \mathrm{W}^{1,1}(\Omega)\right)
$$

and

$$
\mathcal{I}_{F, V, \psi, u}=\int_{0}^{\infty} \int_{\Omega} u(t, x) \partial_{t} \psi(t, x) \mathrm{d} x \mathrm{~d} t+\int_{\Omega} u(0, x) \psi(0, x) \mathrm{d} x
$$

for all $\psi \in \mathrm{C}_{\mathrm{c}}^{2}\left(\mathbb{R} \times \mathbb{R}^{d}\right)$, where $\mathcal{I}_{F, V, \psi, u}$ is defined as

$$
\int_{0}^{\infty} \int_{\Omega}\left[\Lambda\left\langle\nabla L_{F}(u(t, x))+u(t, x) \nabla V(x), \nabla_{x} \psi(t, x)\right\rangle+\Sigma\left(\hat{L}_{F}(u(t, x))+V(x) u(t, x)\right) \psi(t, x)\right] \mathrm{d} x \mathrm{~d} t .
$$


Remark 4.5. $\mathrm{C}_{\mathrm{c}}^{2}\left(\mathbb{R} \times \mathbb{R}^{d}\right)$ denotes the set of all twice continuously differentiable functions $\psi: \mathbb{R} \times \mathbb{R}^{d} \rightarrow \mathbb{R}$ with compact support in $\mathbb{R} \times \mathbb{R}^{d}$. In order to obtain a weak form of the scalar reaction-diffusion equation (1.21), it would suffice to prove (4.14), (4.15) for all twice continuously differentiable functions $\psi: \mathbb{R} \times \Omega \rightarrow \mathbb{R}$ with compact support in $\mathbb{R} \times \Omega$ (short $\psi \in \mathrm{C}_{\mathrm{c}}^{2}(\mathbb{R} \times \Omega)$ ). Establishing (4.14), (4.15) for all $\psi \in \mathrm{C}_{\mathrm{c}}^{2}\left(\mathbb{R} \times \mathbb{R}^{d}\right.$ ) instead means to include the no-flux boundary condition (1.22) in a weak form, and will be an extra challenge in the proof of Theorem 4.4.

Moreover, $\nabla L_{F}(u(t, \cdot)) \in \mathrm{L}^{1}\left(\Omega ; \mathbb{R}^{d}\right)$ denotes the weak gradient of $L_{F}(u(t)) \in \mathrm{W}^{1,1}(\Omega)$, and $\nabla_{x} \psi$ denotes the gradient of $\psi$ with respect to the $x$-variable.

Remark 4.6. By basic convex analysis, $L_{F}$ and $\hat{L}_{F}$ are continuous in $[0,+\infty)$ and $L_{F}$ is nondecreasing, i.e. $L_{F}\left(s_{1}\right) \leq L_{F}\left(s_{2}\right)$ whenever $s_{1} \leq s_{2}$. Furthermore, $L_{F}\left(s_{n}\right) \rightarrow+\infty$ if $s_{n} \rightarrow+\infty$ because $F$ has superlinear growth. Our assumption that $F$ is not only convex but strictly convex seems none too restrictive and makes things considerably easier since in this case, $L_{F}$ is strictly increasing, i.e. $L_{F}\left(s_{1}\right)<L_{F}\left(s_{2}\right)$ whenever $s_{1}<s_{2}$. Thus, we have

$$
L_{F}\left(s_{n}\right) \rightarrow L \in \mathbb{R} \quad \Rightarrow \quad \exists s \in[0,+\infty): L=L_{F}(s), \quad s_{n} \rightarrow s .
$$

The fact that, according to (4.16), $\mathscr{L}^{d}$-a.e.-convergence of $L_{F}\left(u_{n}\right)$ for $u_{n}: \Omega \rightarrow[0,+\infty), u_{n} \in \mathrm{L}^{1}(\Omega)(n \in \mathbb{N})$, translates into $\mathscr{L}^{d}$-a.e. convergence of $u_{n}$ will be a useful ingredient in our proof, $c f$. Section 4.2.

Now, let us be precise about the differentiability condition imposed on $\mathcal{F}$.

Assumption 4.7. We suppose that $L_{F}\left(u_{0}\right) \in \mathrm{L}^{1}(\Omega)$ and

$$
\lim _{h \rightarrow 0} \frac{\mathcal{F}\left(\nu_{h}\right)-\mathcal{F}\left(\nu_{0}\right)}{h}=\int_{\Omega}\left[-L_{F}\left(u_{0}(x)\right) \operatorname{tr} \mathrm{D} v(x)+2 \hat{L}_{F}\left(u_{0}(x)\right) R(x)\right] \mathrm{d} x,
$$

whenever $v: \Omega \rightarrow \mathbb{R}^{d}$ is continuously differentiable and has compact support in $\Omega, R: \Omega \rightarrow \mathbb{R}$ is bounded and Borel measurable, $\nu_{0}=u_{0} \mathscr{L}^{d} \in\{\mathcal{F}<+\infty\}$ and the curve $\mathcal{N} \ni h \mapsto \nu_{h} \in \mathcal{M}(\bar{\Omega})$ is defined according to (3.1), i.e.

$$
\nu_{h}:=(I+h v)_{\#}(1+h R)^{2} \nu_{0}
$$

(where $L_{F}, \hat{L}_{F}$ are defined as in (4.10), $\mathrm{D} v$ denotes the differential of $v$ and $\operatorname{tr} \mathrm{D} v$ its trace).

A similar condition has already been treated in the study of diffusion equations (1.6) (cf. Sect. 10.4.3 in [2]). The differentiability of $h \mapsto \mathcal{F}\left(\nu_{h}\right)$ at $h=0$, for such curves $h \mapsto \nu_{h}$, together with our analysis from Section 3, will form the very basis for proving (4.14), (4.15). We note that, if $\nu_{0}=u_{0} \mathscr{L}^{d}$ and $\nu_{h}$ is defined as above, then,

$$
\nu_{h}=u_{h} \mathscr{L}^{d}, \quad \operatorname{det}(\mathbb{I}+h \mathrm{D} v(x)) u_{h}(x+h v(x))=(1+h R(x))^{2} u_{0}(x), \quad x \in \Omega,
$$

for $h$ in a neighbourhood around 0 (where $\mathbb{I}$ denotes the identity matrix). This follows from the change of variables formula and the fact that, for $|h|$ small enough, $I+h v$ is a diffeomorphism mapping $\Omega$ onto $\Omega$ with $\operatorname{det}(\mathrm{D}(I+h v))=\operatorname{det}(\mathbb{I}+h \mathrm{D} v)>0$. Moreover, for every $x \in \Omega$, the mapping $h \mapsto \operatorname{det}(\mathbb{I}+h \mathrm{D} v(x))$ is differentiable at $h=0$ with derivative equal to $\operatorname{tr} \mathrm{D} v(x)$. By (4.19) and the change of variables formula, we have

$$
\frac{\mathcal{F}\left(\nu_{h}\right)-\mathcal{F}\left(\nu_{0}\right)}{h}=\int_{\Omega} \frac{1}{h}\left[F\left(\frac{(1+h R(x))^{2} u_{0}(x)}{\operatorname{det}(\mathbb{I}+h \mathrm{D} v(x))}\right) \operatorname{det}(\mathbb{I}+h \mathrm{D} v(x))-F\left(u_{0}(x)\right)\right] \mathrm{d} x
$$

if $|h|$ is small and $\nu_{h} \in\{\mathcal{F}<+\infty\}$. It is not difficult to see that the integrands of (4.20) converge pointwise to the integrand of the right-hand side of (4.17) as $h \rightarrow 0$. So if the corresponding integrals also converge (e.g. by dominated convergence theorem or monotone convergence theorem), then (4.17) holds good. 
Example 4.8. We give two examples of functions $F:[0,+\infty) \rightarrow \mathbb{R}$ satisfying the assumptions of Theorem 4.4. The first example is

$$
F(s):= \begin{cases}c_{1} s \log s & \text { if } s \in(0,+\infty), \quad\left(c_{1}>0\right), \\ 0 & \text { if } s=0,\end{cases}
$$

for which Theorem 4.4 yields (4.14), (4.15) with

$$
L_{F}(s)=c_{1} s, \quad \hat{L}_{F}(s)= \begin{cases}c_{1} s+c_{1} s \log s & \text { if } s \in(0,+\infty) \\ 0 & \text { if } s=0\end{cases}
$$

In this case, Assumption 4.7 is established by simplifying the right-hand side of (4.20) to

$$
\int_{\Omega}\left[F\left(u_{0}(x)\right) \frac{(1+h R(x))^{2}-1}{h}+c_{1} u_{0}(x)(1+h R(x))^{2} \frac{\log (1+h R(x))^{2}-\log \operatorname{det}(\mathbb{I}+h \mathrm{D} v(x))}{h}\right] \mathrm{d} x
$$

(using arithmetical rules of the logarithm) and by applying the dominated convergence theorem (note that $\int_{\Omega}\left|F\left(u_{0}(x)\right)\right| \mathrm{d} x<+\infty$ if $\left.\nu_{0}=u_{0} \mathscr{L}^{d} \in\{\mathcal{F}<+\infty\}\right)$.

The second basic example to which Theorem 4.4 is applicable is

$$
F(s):=-c_{1} s^{q}+c_{2} s^{p} \quad\left(c_{1} \geq 0, c_{2}>0, p>1, q \in(0,1)\right)
$$

with

$$
L_{F}(s)=c_{1}(1-q) s^{q}+c_{2}(p-1) s^{p}, \quad \hat{L}_{F}(s)=-c_{1} q s^{q}+c_{2} p s^{p} .
$$

In this case, the right-hand side of (4.20) reads as

$$
\int_{\Omega}\left[-c_{1} u_{0}(x)^{q} \frac{(\operatorname{det}(\mathbb{I}+h \mathrm{D} v(x)))^{1-q}(1+h R(x))^{2 q}-1}{h}+c_{2} u_{0}(x)^{p} \frac{(1+h R(x))^{2 p}(\operatorname{det}(\mathbb{I}+h \mathrm{D} v(x)))^{1-p}-1}{h}\right] \mathrm{d} x,
$$

and again, Assumption 4.7 can be established by using the dominated convergence theorem (note that $\left.\int_{\Omega} u_{0}(x)^{q} \mathrm{~d} x \leq \mathscr{L}^{d}(\Omega)+\int_{\Omega} u_{0}(x) \mathrm{d} x\right)$.

Finally, we remark that the Lipschitz continuity of $V$ appears to be a convenient condition for our purposes since in this case, $V \in \mathrm{C}_{b}^{0}(\bar{\Omega})$ and thus $\mathcal{V}$ (defined as in (1.20)) obviously satisfies Assumptions (A1), (A2), (A3) of Proposition 4.1, and in addition, $V$ is a.e. differentiable with bounded gradient $\nabla V$, and, by dominated convergence theorem,

$$
\lim _{h \rightarrow 0} \frac{\mathcal{V}\left(\nu_{h}\right)-\mathcal{V}\left(\nu_{0}\right)}{h}=\int_{\Omega}[\langle\nabla V(x), v(x)\rangle+2 V(x) R(x)] u_{0}(x) \mathrm{d} x
$$

for every curve $\mathcal{N} \ni h \mapsto \nu_{h}:=(I+h v)_{\#}(1+h R)^{2} \nu_{0} \in \mathcal{M}(\bar{\Omega}), \nu_{0}=u_{0} \mathscr{L}^{d}$, defined according to Assumption 4.7.

\subsection{Proof}

We prove Theorem 4.4.

Proof. If the assumptions of Theorem 4.4 hold, then Lemma 4.2, Lemma 4.3 and the discussion in Section 4.1 show that Proposition 4.1 is applicable to $\Phi$ and $\mathcal{E}:=\mathcal{F}+\mathcal{V}: \mathcal{M}(\bar{\Omega}) \rightarrow(-\infty,+\infty]$ defined as in (1.19) and 
(1.20). Hence, for every $\mu_{0}=u_{0} \mathscr{L}^{d} \in\{\mathcal{E}<+\infty\}$, the set $\operatorname{GMM}\left(\Phi ; \mu_{0}\right)$ is nonempty. So let $\mu \in \operatorname{GMM}\left(\Phi ; \mu_{0}\right)$. There exist a subsequence $\left(\tau_{k}\right)_{k \in \mathbb{N}}, \tau_{k} \downarrow 0$, and discrete solutions $\mu_{\tau_{k}}$ to the associated Minimizing Movement scheme (1.1) converging pointwise to $\mu$, i.e. $\mu_{\tau_{k}}(0)=\mu_{0}$ and

$$
\lim _{k \rightarrow \infty} \mathrm{HK}_{\Lambda, \Sigma}\left(\mu_{\tau_{k}}(t), \mu(t)\right)=0 \quad \text { for all } t \geq 0 .
$$

Every discrete solution $\mu_{\tau_{k}}$ is assigned a curve $u_{\tau_{k}}:[0,+\infty) \rightarrow \mathrm{L}^{1}(\Omega)$ such that $u_{\tau_{k}}(0)=u_{0}, u_{\tau_{k}}(t, \cdot) \geq 0$ and

$$
\mu_{\tau_{k}}(t)=u_{\tau_{k}}(t) \mathscr{L}^{d} \quad \text { for all } t \geq 0
$$

We note that, by (1.1), $t \mapsto \mathcal{E}\left(\mu_{\tau_{k}}(t)\right)$ is decreasing. Since $V \in \mathrm{C}_{b}^{0}(\bar{\Omega})$ and $\sup _{k} \mathrm{HK}_{\Lambda, \Sigma}\left(\mu_{\tau_{k}}(t), \eta_{0}\right)<+\infty$, it follows from $\mathcal{E}\left(\mu_{\tau_{k}}(t)\right) \leq \mathcal{E}\left(\mu_{0}\right)$ that $\sup _{k} \mathcal{F}\left(\mu_{\tau_{k}}(t)\right)<+\infty$. Thus, according to Lemma 4.3, there exists a curve $u:[0,+\infty) \rightarrow \mathrm{L}^{1}(\Omega)$ such that $u(0)=u_{0}, u(t, \cdot) \geq 0$ and

$$
u_{\tau_{k}}(t) \stackrel{\mathrm{L}^{1}}{\rightarrow} u(t), \quad \mu(t)=u(t) \mathscr{L}^{d} \quad \text { for all } t \geq 0
$$

(this convergence holds good for the whole sequence $\tau_{k} \downarrow 0$ as we already know that $\left.\mathrm{HK}_{\Lambda, \Sigma}\left(\mu_{\tau_{k}}(t), \mu(t)\right) \rightarrow 0\right)$. By Proposition 4.1, $\mu$ is continuous, i.e. $\mathrm{HK}_{\Lambda, \Sigma}\left(\mu\left(t_{n}\right), \mu(t)\right) \rightarrow 0$ whenever $t_{n} \rightarrow t$. Since $\mathcal{E}(\mu(t)) \leq \mathcal{E}\left(\mu_{0}\right)$ for all $t \geq 0$, the same arguments as before show that

$$
u\left(t_{n}\right) \stackrel{\mathrm{L}^{1}}{\rightarrow} u(t) \quad \text { whenever } t_{n} \rightarrow t, t \geq 0 .
$$

Now, let $v: \Omega \rightarrow \mathbb{R}^{d}$ be a continuously differentiable function with compact support in $\Omega$ and let $R: \Omega \rightarrow \mathbb{R}$ be a bounded Borel measurable function. We define, for $k \in \mathbb{N}, n \in \mathbb{N}$, the curve

$$
\mathcal{N} \ni h \mapsto \nu_{h}:=(I+h v)_{\#}(1+h R)^{2} \mu_{\tau_{k}}\left(n \tau_{k}\right) \in \mathcal{M}(\bar{\Omega})
$$

according to (3.1). We recall that

$$
\mu_{\tau_{k}}\left(n \tau_{k}\right) \text { is a minimizer for } \Phi\left(\tau_{k}, \mu_{\tau_{k}}\left((n-1) \tau_{k}\right), \cdot\right)=\mathcal{E}(\cdot)+\frac{1}{2 \tau_{k}} \mathbf{H}_{\Lambda, \Sigma}\left(\cdot, \mu_{\tau_{k}}\left((n-1) \tau_{k}\right)\right)^{2},
$$

and we establish a necessary condition of first order involving the Fréchet subdifferential of

$$
h \mapsto-\frac{1}{2} \mathrm{HK}_{\Lambda, \Sigma}\left(\nu_{h}, \mu_{\tau_{k}}\left((n-1) \tau_{k}\right)\right)^{2}
$$

at $h=0$ and the directional derivatives (4.17) and (4.21) of $\mathcal{F}$ and $\mathcal{V}$. We set $\mu_{\tau_{k}}^{n}:=\mu_{\tau_{k}}\left(n \tau_{k}\right), u_{\tau_{k}}^{n}:=u_{\tau_{k}}\left(n \tau_{k}\right)$. Let $\beta_{\tau_{k}}^{n} \in \mathcal{M}(\mathfrak{C} \times \mathfrak{C})$ be optimal in the definition of $\mathrm{H}_{\Lambda, \Sigma}\left(\mu_{\tau_{k}}^{n}, \mu_{\tau_{k}}^{n-1}\right)^{2}$ according to (2.6), (2.4), with first marginal $\alpha_{\tau_{k}}^{n} \in \mathcal{M}_{2}(\mathfrak{C}), \mathfrak{h} \alpha_{\tau_{k}}^{n}=\mu_{\tau_{k}}^{n}$, and second marginal $\alpha_{\tau_{k}}^{n-1}, \mathfrak{h} \alpha_{\tau_{k}}^{n-1}=\mu_{\tau_{k}}^{n-1}$. Since $\mu_{\tau_{k}}^{n}$ is a minimizer for $\Phi\left(\tau_{k}, \mu_{\tau_{k}}^{n-1}, \cdot\right)$, we have

$$
\frac{\mathcal{E}\left(\nu_{h}\right)-\mathcal{E}\left(\mu_{\tau_{k}}^{n}\right)}{h} \geq \frac{\frac{1}{2 \tau_{k}} \mathrm{H}_{\Lambda, \Sigma}\left(\mu_{\tau_{k}}^{n}, \mu_{\tau_{k}}^{n-1}\right)^{2}-\frac{1}{2 \tau_{k}} \mathrm{HK}_{\Lambda, \Sigma}\left(\nu_{h}, \mu_{\tau_{k}}^{n-1}\right)^{2}}{h}, \quad h \in \mathcal{N}, h>0,
$$

and passing to the limit $h \downarrow 0$, we obtain

$$
\int_{\Omega}\left[-L_{F}\left(u_{\tau_{k}}^{n}(x)\right) \operatorname{tr} \mathrm{D} v(x)+2 \hat{L}_{F}\left(u_{\tau_{k}}^{n}(x)\right) R(x)+(\langle\nabla V(x), v(x)\rangle+2 V(x) R(x)) u_{\tau_{k}}^{n}(x)\right] \mathrm{d} x \geq \frac{1}{\tau_{k}} \mathfrak{F}_{\tau_{k}, n, v, R},
$$


with

$$
\begin{aligned}
& \mathfrak{F}_{\tau_{k}, n, v, R}:=\frac{4}{\Sigma} \int_{\mathfrak{C} \times \mathfrak{C}}\left[-r_{1}^{2} R\left(x_{1}\right)+r_{1} r_{2} R\left(x_{1}\right) \cos \left(\sqrt{\Sigma / 4 \Lambda}\left|x_{1}-x_{2}\right|\right)+r_{1} r_{2} \sqrt{\Sigma / 4 \Lambda}\left\langle S_{\Lambda, \Sigma}\left(x_{1}, x_{2}\right), v\left(x_{1}\right)\right\rangle\right] \mathrm{d} \beta_{\tau_{k}}^{n}, \\
& S_{\Lambda, \Sigma}\left(x_{1}, x_{2}\right):= \begin{cases}\frac{\sin \left(\sqrt{\Sigma / 4 \Lambda}\left|x_{1}-x_{2}\right|\right)}{\left|x_{1}-x_{2}\right|}\left(x_{2}-x_{1}\right) & \text { if } x_{1} \neq x_{2}, \\
0 & \text { if } x_{1}=x_{2},\end{cases}
\end{aligned}
$$

by Proposition 3.3, Assumption 4.7 and (4.21). As we can switch between $v, R$ and $-v,-R$, the following necessary condition of first order holds good

$$
\int_{\Omega}\left[-L_{F}\left(u_{\tau_{k}}^{n}\right) \operatorname{tr} \mathrm{D} v+2 \hat{L}_{F}\left(u_{\tau_{k}}^{n}\right) R+(\langle\nabla V, v\rangle+2 V R) u_{\tau_{k}}^{n}\right] \mathrm{d} x=\frac{1}{\tau_{k}} \mathfrak{F}_{\tau_{k}, n, v, R}
$$

for all continuously differentiable functions $v: \Omega \rightarrow \mathbb{R}^{d}$ with compact support in $\Omega$ and all bounded Borel measurable functions $R: \Omega \rightarrow \mathbb{R}$.

Let $\phi: \Omega \rightarrow \mathbb{R}$ be a twice continuously differentiable function with compact support in $\Omega$. Setting $v:=\frac{4 \Lambda}{\Sigma} \nabla \phi$, $R:=2 \phi$ and applying our necessary condition of first order (4.22) and Proposition 3.5, we obtain

$$
\begin{aligned}
& \quad\left|\int_{\Omega}\left[-\Lambda L_{F}\left(u_{\tau_{k}}^{n}\right) \Delta \phi+\Sigma \hat{L}_{F}\left(u_{\tau_{k}}^{n}\right) \phi+(\Lambda\langle\nabla V, \nabla \phi\rangle+\Sigma V \phi) u_{\tau_{k}}^{n}\right] \mathrm{d} x-\frac{1}{\tau_{k}}\left(\int_{\Omega} \phi \mathrm{d} \mu_{\tau_{k}}^{n-1}-\int_{\Omega} \phi \mathrm{d} \mu_{\tau_{k}}^{n}\right)\right| \\
& \leq \frac{1}{\tau_{k}} C_{\phi, \Lambda, \Sigma} \mathrm{HK}_{\Lambda, \Sigma}\left(\mu_{\tau_{k}}^{n}, \mu_{\tau_{k}}^{n-1}\right)^{2},
\end{aligned}
$$

with $C_{\phi, \Lambda, \Sigma} \geq 0$ only depending on $\phi, \Lambda, \Sigma$ (cf. (3.12), (3.13)). Hence, for every $\psi \in \mathrm{C}_{\mathrm{c}}^{2}(\mathbb{R} \times \Omega)$ there exist $C_{\psi, \Lambda, \Sigma}>0, N_{\psi, \tau_{k}} \in \mathbb{N}$ such that $\psi(t, \cdot) \equiv 0$ for $t \geq N_{\psi, \tau_{k}} \tau_{k}, \sup _{k} N_{\psi, \tau_{k}} \tau_{k}<+\infty$ and

$$
\begin{aligned}
& \left|\int_{0}^{\infty} \mathfrak{I}_{\tau_{k}, \psi}(t) \mathrm{d} t-\int_{\tau_{k}}^{\infty} \int_{\Omega} \frac{u_{\tau_{k}}\left(t-\tau_{k}, x\right)-u_{\tau_{k}}(t, x)}{\tau_{k}} \psi(t, x) \mathrm{d} x \mathrm{~d} t-\int_{0}^{\tau_{k}} \int_{\Omega} \frac{u_{\tau_{k}}(0, x)-u_{\tau_{k}}(t, x)}{\tau_{k}} \psi(t, x) \mathrm{d} x \mathrm{~d} t\right| \\
& \leq C_{\psi, \Lambda, \Sigma} \sum_{n=1}^{N_{\psi, \tau_{k}}} \mathrm{HK}_{\Lambda, \Sigma}\left(\mu_{\tau_{k}}^{n}, \mu_{\tau_{k}}^{n-1}\right)^{2},
\end{aligned}
$$

where $\mathfrak{I}_{\tau_{k}, \psi}(t)$ is defined as

$$
\int_{\Omega}\left[-\Lambda L_{F}\left(u_{\tau_{k}}(t, x)\right) \Delta_{x} \psi(t, x)+\Sigma \hat{L}_{F}\left(u_{\tau_{k}}(t, x)\right) \psi(t, x)+\left(\Lambda\left\langle\nabla V(x), \nabla_{x} \psi(t, x)\right\rangle+\Sigma V(x) \psi(t, x)\right) u_{\tau_{k}}(t, x)\right] \mathrm{d} x .
$$

Standard tools from the theory of the Minimizing Movement approach to gradient flows yield

$$
\begin{aligned}
& \sup \left\{\mathrm{HK}_{\Lambda, \Sigma}\left(\mu_{\tau_{k}}^{n}, \eta_{0}\right)^{2}: 1 \leq n \leq N_{k}, k \in \mathbb{N}\right\}<+\infty, \\
& \sum_{n=1}^{N_{k}} \mathrm{HK}_{\Lambda, \Sigma}\left(\mu_{\tau_{k}}^{n}, \mu_{\tau_{k}}^{n-1}\right)^{2} \leq 2 \tau_{k}\left(\mathcal{E}\left(\mu_{0}\right)+A+B \mathrm{H}_{\Lambda, \Sigma}\left(\mu_{\tau_{k}}^{N_{k}}, \eta_{0}\right)^{2}\right) \rightarrow 0 \quad \text { as } k \rightarrow \infty,
\end{aligned}
$$

whenever $\sup _{k} N_{k} \tau_{k}<+\infty$ (and where $A, B>0$ s.t. $\mathcal{E}(\cdot) \geq-A-B \mathrm{HK}_{\Lambda, \Sigma}\left(\cdot, \eta_{0}\right)^{2}$ ), see e.g. the first part of the proof in [10], Section 3.2. Furthermore, we have

$$
\int_{\tau_{k}}^{\infty} \int_{\Omega} \frac{u_{\tau_{k}}\left(t-\tau_{k}, x\right)-u_{\tau_{k}}(t, x)}{\tau_{k}} \psi(t, x) \mathrm{d} x \mathrm{~d} t+\int_{0}^{\tau_{k}} \int_{\Omega} \frac{u_{\tau_{k}}(0, x)-u_{\tau_{k}}(t, x)}{\tau_{k}} \psi(t, x) \mathrm{d} x \mathrm{~d} t
$$




$$
\begin{aligned}
& =\int_{0}^{\infty} \int_{\Omega} \frac{\psi\left(t+\tau_{k}, x\right)-\psi(t, x)}{\tau_{k}} u_{\tau_{k}}(t, x) \mathrm{d} x \mathrm{~d} t+\frac{1}{\tau_{k}} \int_{0}^{\tau_{k}} \int_{\Omega} u_{\tau_{k}}(0, x) \psi(t, x) \mathrm{d} x \mathrm{~d} t \\
& \rightarrow \int_{0}^{\infty} \int_{\Omega} u(t, x) \partial_{t} \psi(t, x) \mathrm{d} x \mathrm{~d} t+\int_{\Omega} u(0, x) \psi(0, x) \mathrm{d} x \quad \text { as } \tau_{k} \downarrow 0
\end{aligned}
$$

(since $\partial_{t} \psi$ is uniformly continuous and bounded, $u_{\tau_{k}}(t) \stackrel{\mathrm{L}^{1}}{\rightarrow} u(t), \sup \left\{\int_{\Omega} u_{\tau_{k}}(t, x) \mathrm{d} x: t \leq N_{\psi, \tau_{k}} \tau_{k}, k \in \mathbb{N}\right\}=$ $\left.\sup \left\{\frac{\Sigma}{4} \mathrm{HK}_{\Lambda, \Sigma}\left(\mu_{\tau_{k}}(t), \eta_{0}\right)^{2}: t \leq N_{\psi, \tau_{k}} \tau_{k}, k \in \mathbb{N}\right\}<+\infty\right)$. So in order to establish (4.14) for $\psi \in \mathrm{C}_{\mathrm{c}}^{2}(\mathbb{R} \times \Omega)$, all that remains is to prove that $L_{F}(u(t)) \in \mathrm{W}^{1,1}(\Omega)$ for a.e. $t>0$ and $\int_{0}^{\infty} \mathfrak{I}_{\tau_{k}, \psi}(t) \mathrm{d} t \rightarrow \mathcal{I}_{F, V, \psi, u}$ as $\tau_{k} \downarrow 0$. Again, the necessary condition of first order will smooth the way. We set $R \equiv 0$ in (4.22) and obtain

$$
\begin{aligned}
& \left|\int_{\Omega}-L_{F}\left(u_{\tau_{k}}^{n}(x)\right) \operatorname{tr} \mathrm{D} v(x) \mathrm{d} x\right| \\
& \leq \sup _{x \in \Omega}|\nabla V(x)| \int_{\Omega}|v(x)| u_{\tau_{k}}^{n}(x) \mathrm{d} x+\frac{1}{\tau_{k}}\left(\int_{\mathfrak{C} \times \mathfrak{C}} \frac{4}{\Sigma \Lambda} r_{2}^{2}\left|S_{\Lambda, \Sigma}\left(x_{1}, x_{2}\right)\right|^{2} \mathrm{~d} \beta_{\tau_{k}}^{n}\right)^{1 / 2}\left(\int_{\mathfrak{C} \times \mathfrak{C}} r_{1}^{2}\left|v\left(x_{1}\right)\right|^{2} \mathrm{~d} \beta_{\tau_{k}}^{n}\right)^{1 / 2} \\
& \leq\left(\sup _{x \in \Omega}|\nabla V(x)|\left(\int_{\Omega} u_{\tau_{k}}^{n}(x) \mathrm{d} x\right)^{1 / 2}+\frac{1}{\sqrt{\Lambda}} \frac{\mathrm{HK}_{\Lambda, \Sigma}\left(\mu_{\tau_{k}}^{n}, \mu_{\tau_{k}}^{n-1}\right)}{\tau_{k}}\right)\left(\int_{\Omega}|v(x)|^{2} u_{\tau_{k}}^{n}(x) \mathrm{d} x\right)^{1 / 2} \\
& \leq\left(\sup _{x \in \Omega}|\nabla V(x)|\left(\int_{\Omega} u_{\tau_{k}}^{n}(x) \mathrm{d} x\right)^{1 / 2}+\frac{1}{\sqrt{\Lambda}} \frac{\mathrm{HK}_{\Lambda, \Sigma}\left(\mu_{\tau_{k}}^{n}, \mu_{\tau_{k}}^{n-1}\right)}{\tau_{k}}\right)\left(\int_{\Omega} u_{\tau_{k}}^{n}(x) \mathrm{d} x\right)^{1 / 2} \sup _{x \in \Omega}|v(x)|
\end{aligned}
$$

by applying Hölder's inequality and (2.14) and (2.15) which yield

$$
\int_{\mathfrak{C} \times \mathfrak{C}} \frac{4}{\Sigma} r_{2}^{2}\left|S_{\Lambda, \Sigma}\left(x_{1}, x_{2}\right)\right|^{2} \mathrm{~d} \beta_{\tau_{k}}^{n} \leq \int_{\mathfrak{C} \times \mathfrak{C}} \mathrm{d}_{\mathfrak{C}, \Lambda, \Sigma}\left(\left[x_{1}, r_{1}\right],\left[x_{2}, r_{2}\right]\right)^{2} \mathrm{~d} \beta_{\tau_{k}}^{n}=\mathrm{HK}_{\Lambda, \Sigma}\left(\mu_{\tau_{k}}^{n}, \mu_{\tau_{k}}^{n-1}\right)^{2}
$$

(note that (2.14) holds good for $t=0$, too, as $\theta_{+}^{\prime}(0)=\lim _{t \downarrow 0} \theta^{\prime}(t)$ and $\mathcal{R}_{+}^{\prime}(0)=\lim _{t \downarrow 0} \mathcal{R}^{\prime}(t)$ ). Firstly, the above estimations (which hold for every continuously differentiable function $v: \Omega \rightarrow \mathbb{R}^{d}$ with compact support in $\Omega$ ) show that $L_{F}\left(u_{\tau_{k}}^{n}\right)$ is a function of bounded variation in $\Omega$ (recall that $L_{F}\left(u_{\tau_{k}}^{n}\right) \in \mathrm{L}^{1}(\Omega)$ by Assumption 4.7), and in a second step, they show, according to Hahn-Banach Theorem and the fact that the dual space of $\mathrm{L}^{2}\left(\Omega ; u_{\tau_{k}}^{n} \mathscr{L}^{d}\right)$ can be identified with $\mathrm{L}^{2}\left(\Omega ; u_{\tau_{k}}^{n} \mathscr{L}^{d}\right)$ itself, the existence of a function $w_{\tau_{k}}^{n}: \Omega \rightarrow \mathbb{R}^{d}$ such that

$$
\begin{aligned}
\int_{\Omega}\left|w_{\tau_{k}}^{n}(x)\right|^{2} u_{\tau_{k}}^{n}(x) \mathrm{d} x & \leq\left(\sup _{x \in \Omega}|\nabla V(x)|\left(\int_{\Omega} u_{\tau_{k}}^{n}(x) \mathrm{d} x\right)^{1 / 2}+\frac{1}{\sqrt{\Lambda}} \frac{H \mathrm{~K}_{\Lambda, \Sigma}\left(\mu_{\tau_{k}}^{n}, \mu_{\tau_{k}}^{n-1}\right)}{\tau_{k}}\right)^{2} \\
\int_{\Omega}-L_{F}\left(u_{\tau_{k}}^{n}(x)\right) \operatorname{tr} \mathrm{D} v(x) \mathrm{d} x & =\int_{\Omega}\left\langle w_{\tau_{k}}^{n}(x), v(x)\right\rangle u_{\tau_{k}}^{n}(x) \mathrm{d} x
\end{aligned}
$$

for every continuously differentiable function $v: \Omega \rightarrow \mathbb{R}^{d}$ with compact support in $\Omega$. This means that $L_{F}\left(u_{\tau_{k}}^{n}\right) \in$ $\mathrm{W}^{1,1}(\Omega)$ with weak gradient $\nabla L_{F}\left(u_{\tau_{k}}^{n}\right)=w_{\tau_{k}}^{n} u_{\tau_{k}}^{n}$ and

$$
\int_{\Omega}\left|\nabla L_{F}\left(u_{\tau_{k}}^{n}(x)\right)\right| \mathrm{d} x \leq\left(\sup _{x \in \Omega}|\nabla V(x)|\left(\int_{\Omega} u_{\tau_{k}}^{n}(x) \mathrm{d} x\right)^{1 / 2}+\frac{1}{\sqrt{\Lambda}} \frac{\mathrm{K}_{\Lambda, \Sigma}\left(\mu_{\tau_{k}}^{n}, \mu_{\tau_{k}}^{n-1}\right)}{\tau_{k}}\right)\left(\int_{\Omega} u_{\tau_{k}}^{n}(x) \mathrm{d} x\right)^{1 / 2} .
$$

Now, let us give an upper bound for $L_{F}\left(u_{\tau_{k}}^{n}\right)$ in $\mathrm{L}^{1}(\Omega)$ so that in the end, we have an upper bound for $L_{F}\left(u_{\tau_{k}}^{n}\right)$ in $\mathrm{W}^{1,1}(\Omega)$. We note that

$$
\int_{\Omega}\left|L_{F}\left(u_{\tau_{k}}^{n}\right)\right| \mathrm{d} x \leq \int_{\Omega}\left(\left|\hat{L}_{F}\left(u_{\tau_{k}}^{n}\right)\right|+\left|F\left(u_{\tau_{k}}^{n}\right)\right|\right) \mathrm{d} x \leq \int_{\Omega}\left|\hat{L}_{F}\left(u_{\tau_{k}}^{n}\right)\right| \mathrm{d} x+\mathcal{F}\left(\mu_{\tau_{k}}^{n}\right)+2 \int_{\Omega}\left[\left|F(1)-F^{\prime}(1)\right|+\left|F^{\prime}(1)\right| u_{\tau_{k}}^{n}\right] \mathrm{d} x
$$


and setting $v \equiv 0$ in (4.22), we obtain

$$
\begin{aligned}
\left|\int_{\Omega} \hat{L}_{F}\left(u_{\tau_{k}}^{n}(x)\right) R(x) \mathrm{d} x\right| \leq & \left(\sup _{x \in \Omega}|V(x)| \int_{\Omega} u_{\tau_{k}}^{n}(x) \mathrm{d} x\right) \sup _{x \in \Omega}|R(x)| \\
& +\frac{2}{\tau_{k} \Sigma}\left(\int_{\mathfrak{C} \times \mathfrak{C}} Q_{\Lambda, \Sigma}\left(x_{1}, r_{1}, x_{2}, r_{2}\right)^{2} \mathrm{~d} \beta_{\tau_{k}}^{n}\right)^{1 / 2}\left(\int_{\Omega} u_{\tau_{k}}^{n}(x) \mathrm{d} x\right)^{1 / 2} \sup _{x \in \Omega}|R(x)|
\end{aligned}
$$

for all bounded Borel functions $R: \Omega \rightarrow \mathbb{R}$, with $Q_{\Lambda, \Sigma}\left(\left[x_{1}, r_{1}\right],\left[x_{2}, r_{2}\right]\right):=-r_{1}+r_{2} \cos \left(\sqrt{\Sigma / 4 \Lambda}\left|x_{1}-x_{2}\right|\right)$ and

$$
\int_{\mathfrak{C} \times \mathfrak{C}} Q_{\Lambda, \Sigma}\left(\left[x_{1}, r_{1}\right],\left[x_{2}, r_{2}\right]\right)^{2} \mathrm{~d} \beta_{\tau_{k}}^{n} \leq \frac{\Sigma}{4} \int_{\mathfrak{C} \times \mathfrak{C}} \mathrm{d}_{\mathfrak{C}, \Lambda, \Sigma}\left(\left[x_{1}, r_{1}\right],\left[x_{2}, r_{2}\right]\right)^{2} \mathrm{~d} \beta_{\tau_{k}}^{n}=\frac{\Sigma}{4} \mathrm{HK}_{\Lambda, \Sigma}\left(\mu_{\tau_{k}}^{n}, \mu_{\tau_{k}}^{n-1}\right)^{2}
$$

by (2.14) (which also holds good at $t=0)$ and (2.15). Hence,

$$
\int_{\Omega}\left|\hat{L}_{F}\left(u_{\tau_{k}}^{n}(x)\right)\right| \mathrm{d} x \leq \sup _{x \in \Omega}|V(x)| \int_{\Omega} u_{\tau_{k}}^{n}(x) \mathrm{d} x+\frac{1}{\sqrt{\Sigma}} \frac{\mathrm{K}_{\Lambda, \Sigma}\left(\mu_{\tau_{k}}^{n}, \mu_{\tau_{k}}^{n-1}\right)}{\tau_{k}}\left(\int_{\Omega} u_{\tau_{k}}^{n}(x) \mathrm{d} x\right)^{1 / 2} .
$$

Similarly, we obtain

$$
\int_{E}\left|\hat{L}_{F}\left(u_{\tau_{k}}^{n}(x)\right)\right| \mathrm{d} x \leq \sup _{x \in \Omega}|V(x)| \int_{E} u_{\tau_{k}}^{n}(x) \mathrm{d} x+\frac{1}{\sqrt{\Sigma}} \frac{\mathrm{HK}_{\Lambda, \Sigma}\left(\mu_{\tau_{k}}^{n}, \mu_{\tau_{k}}^{n-1}\right)}{\tau_{k}}\left(\int_{E} u_{\tau_{k}}^{n}(x) \mathrm{d} x\right)^{1 / 2}
$$

for every Borel set $E \subset \Omega$. Define $\left|\mu_{\tau_{k}}^{\prime}\right|:(0,+\infty) \rightarrow[0,+\infty)$ as

$$
\left|\mu_{\tau_{k}}^{\prime}\right|(t):=\frac{\mathrm{K}_{\Lambda, \Sigma}\left(\mu_{\tau_{k}}^{n}, \mu_{\tau_{k}}^{n-1}\right)}{\tau_{k}} \quad \text { for } t \in\left((n-1) \tau_{k}, n \tau_{k}\right] \quad(n \in \mathbb{N})
$$

We have found out so far that $L_{F}\left(u_{\tau_{k}}(t)\right) \in \mathrm{W}^{1,1}(\Omega)$ for all $t>0$ and $k \in \mathbb{N}$ and that, if $\sup _{l}\left|\mu_{\tau_{k_{l}}}^{\prime}\right|(t)<+\infty$ for some subsequence $\left(\tau_{k_{l}}\right)_{l \in \mathbb{N}}, \tau_{k_{l}} \downarrow 0$, and $t>0$, then $\left(L_{F}\left(u_{\tau_{k_{l}}}(t)\right)\right)_{l \in \mathbb{N}}$ is bounded in $\mathrm{W}^{1,1}(\Omega)$. In this case, by Rellich-Kondrachov Theorem, there exists a subsequence of $\left(L_{F}\left(u_{\tau_{k_{l}}}(t)\right)\right)_{l \in \mathbb{N}}$ which converges strongly in $\mathrm{L}^{1}(\Omega)$. If $\left(L_{F}\left(u_{\tau_{k_{l_{j}}}}(t)\right)\right)_{j \in \mathbb{N}}$ converges to some $\mathfrak{L} \in \mathrm{L}^{1}(\Omega)$, then it will, in turn, contain a subsequence which converges to $\mathfrak{L}$ pointwise $\mathscr{L}^{d}$-a.e., and by (4.16), the corresponding subsequence of $\left(u_{\tau_{k_{l_{j}}}}(t)\right)_{j \in \mathbb{N}}$ will converge to some $\mathfrak{u}: \Omega \rightarrow[0,+\infty)$ pointwise $\mathscr{L}^{d}$-a.e. and $\mathfrak{L}=L_{F}(\mathfrak{u})$. Using Egorov Theorem and the facts that $u_{\tau_{k}}(t) \stackrel{\mathrm{L}^{1}}{\rightarrow} u(t)$ and $\mathfrak{u} \in \mathrm{L}^{1}(\Omega)$ by Fatou's lemma, we obtain $\mathfrak{u}=u(t)$ and thus $\mathfrak{L}=L_{F}(u(t))$. This shows that the whole sequence $\left(L_{F}\left(u_{\tau_{k_{l}}}(t)\right)\right)_{l \in \mathbb{N}}$ converges to $L_{F}(u(t))$ strongly in $L^{1}(\Omega)$ whenever $\sup _{l}\left|\mu_{\tau_{k_{l}}}^{\prime}\right|(t)<+\infty$. Furthermore, in this case, the corresponding sequence $\left(\nabla L_{F}\left(u_{\tau_{k_{l}}}(t)\right)\right)_{l \in \mathbb{N}}$ of weak gradients is bounded in $\mathrm{L}^{1}\left(\Omega ; \mathbb{R}^{d}\right)$ and equiintegrable because $\left(u_{\tau_{k_{l}}}(t)\right)_{l \in \mathbb{N}}$ is equiintegrable and

$$
\int_{E}\left|\nabla L_{F}\left(u_{\tau_{k_{l}}}(t, x)\right)\right| \mathrm{d} x \leq\left(\sup _{x \in \Omega}|\nabla V(x)|\left(\int_{\Omega} u_{\tau_{k_{l}}}(t, x) \mathrm{d} x\right)^{1 / 2}+\frac{1}{\sqrt{\Lambda}}\left|\mu_{\tau_{k_{l}}}^{\prime}\right|(t)\right)\left(\int_{E} u_{\tau_{k_{l}}}(t, x) \mathrm{d} x\right)^{1 / 2}
$$

for every Borel set $E \subset \Omega$, by the preceding estimations of $\int_{\Omega}\left|w_{\tau_{k}}^{n}(x)\right|^{2} u_{\tau_{k}}^{n}(x) \mathrm{d} x, \nabla L_{F}\left(u_{\tau_{k}}^{n}\right)=w_{\tau_{k}}^{n} u_{\tau_{k}}^{n}$ and Hölder's inequality. Dunford-Pettis Theorem and the above considerations show that $L_{F}(u(t)) \in \mathrm{W}^{1,1}(\Omega)$ for every $t>0$ for which there exists a bounded subsequence of $\left(\left|\mu_{\tau_{k}}^{\prime}\right|(t)\right)_{k \in \mathbb{N}}$ and $\left(\nabla L_{F}\left(u_{\tau_{k_{l}}}(t)\right)\right)_{l \in \mathbb{N}}$ converges to $\nabla L_{F}(u(t))$ weakly in $\mathrm{L}^{1}\left(\Omega ; \mathbb{R}^{d}\right)$ whenever $\sup _{l}\left|\mu_{\tau_{k_{l}}}^{\prime}\right|(t)<+\infty$. By Fatou's lemma, this is true for a.e. $t>0$ 
because (4.23), (4.24) yield

$$
\Delta_{T}:=\sup _{k \in \mathbb{N}} \int_{0}^{T}\left|\mu_{\tau_{k}}^{\prime}\right|(t)^{2} \mathrm{~d} t<+\infty \quad \text { for all } T>0
$$

Now, let $t>0$ and $\tau_{k_{l}} \downarrow 0$ such that $\sup _{l}\left|\mu_{\tau_{k_{l}}}^{\prime}\right|(t)<+\infty$. Using Dunford-Pettis-Theorem, the above estimation of $\int_{E}\left|\hat{L}_{F}\left(u_{\tau_{k}}^{n}(x)\right)\right| \mathrm{d} x$ and the equiintegrability of $\left(u_{\tau_{k_{l}}}(t)\right)_{l \in \mathbb{N}}$, we see that there exists a subsequence of $\left(\hat{L}_{F}\left(u_{\tau_{k_{l}}}(t)\right)\right)_{l \in \mathbb{N}}$ which converges weakly in $\mathrm{L}^{1}(\Omega)$. The preceding considerations show that every subsequence of $\left(u_{\tau_{k_{l}}}\right)_{l \in \mathbb{N}}$ contains a subsubsequence which converges to $u(t)$ pointwise $\mathscr{L}^{d}$-a.e.. We may use Egorov Theorem and the continuity of $\hat{L}_{F}$ in order to conclude that the whole sequence $\left(\hat{L}_{F}\left(u_{\tau_{k_{l}}}(t)\right)\right)_{l \in \mathbb{N}}$ converges to $\hat{L}_{F}(u(t))$ weakly in $\mathrm{L}^{1}(\Omega)$. It is apparent from (4.25) and the preceding convergence results and estimations that $L_{F}(u) \in \mathrm{L}_{\text {loc }}^{2}\left([0,+\infty) ; \mathrm{W}^{1,1}(\Omega)\right)$ and $\hat{L}_{F}(u) \in \mathrm{L}_{\text {loc }}^{2}\left([0,+\infty) ; \mathrm{L}^{1}(\Omega)\right)$.

All in all, we obtain

$$
\begin{aligned}
& \liminf _{k \rightarrow \infty}\left[\mathfrak{I}_{\tau_{k}, \psi}(t)+\epsilon\left|\mu_{\tau_{k}}^{\prime}\right|(t)^{2}\right] \\
& \geq \int_{\Omega}\left[\Lambda\left\langle\nabla L_{F}(u(t, x))+u(t, x) \nabla V(x), \nabla_{x} \psi(t, x)\right\rangle+\Sigma\left(\hat{L}_{F}(u(t, x))+V(x) u(t, x)\right) \psi(t, x)\right] \mathrm{d} x
\end{aligned}
$$

for every $\psi \in \mathrm{C}_{\mathrm{c}}^{2}(\mathbb{R} \times \Omega), \epsilon>0$ and almost every $t>0$, where $\mathfrak{I}_{\tau_{k}, \psi}(t)$ is defined as above, i.e. as

$$
\int_{\Omega}\left[-\Lambda L_{F}\left(u_{\tau_{k}}(t, x)\right) \Delta_{x} \psi(t, x)+\Sigma \hat{L}_{F}\left(u_{\tau_{k}}(t, x)\right) \psi(t, x)+\left(\Lambda\left\langle\nabla V(x), \nabla_{x} \psi(t, x)\right\rangle+\Sigma V(x) \psi(t, x)\right) u_{\tau_{k}}(t, x)\right] \mathrm{d} x
$$

Note that, by the above estimations of $\int_{\Omega}\left|\hat{L}_{F}\left(u_{\tau_{k}}^{n}(x)\right)\right| \mathrm{d} x$ and $\left|\int_{\Omega}-L_{F}\left(u_{\tau_{k}}^{n}(x)\right) \operatorname{tr} \mathrm{D} v(x) \mathrm{d} x\right|$ (here we set $v(x)$ equal to $\left.\nabla_{x} \psi(t, x)\right)$ and by Cauchy's inequality with $\epsilon>0$, we have

$$
\begin{aligned}
& \mathfrak{I}_{\tau_{k}, \psi}(t)+\epsilon\left|\mu_{\tau_{k}}^{\prime}\right|(t)^{2} \\
& \geq-\Lambda\left(C_{V} C_{T_{\psi}}^{1 / 2}+\frac{1}{\sqrt{\Lambda}}\left|\mu_{\tau_{k}}^{\prime}\right|(t)\right) C_{T_{\psi}}^{1 / 2} C_{\psi}-\Sigma C_{\psi}\left(C_{V} C_{T_{\psi}}+\frac{1}{\sqrt{\Sigma}}\left|\mu_{\tau_{k}}^{\prime}\right|(t) C_{T_{\psi}}^{1 / 2}\right)-(\Lambda+\Sigma) C_{V} C_{\psi} C_{T_{\psi}}+\epsilon\left|\mu_{\tau_{k}}^{\prime}\right|(t)^{2} \\
& \geq-2(\Lambda+\Sigma) C_{V} C_{T_{\psi}} C_{\psi}-\sqrt{\Lambda}\left|\mu_{\tau_{k}}^{\prime}\right|(t) C_{T_{\psi}}^{1 / 2} C_{\psi}-\sqrt{\Sigma}\left|\mu_{\tau_{k}}\right|^{\prime}(t) C_{T_{\psi}}^{1 / 2} C_{\psi}+\epsilon\left|\mu_{\tau_{k}}^{\prime}\right|(t)^{2} \\
& \geq-(\Lambda+\Sigma)\left(2 C_{V} C_{T_{\psi}} C_{\psi}+\frac{1}{\epsilon} C_{T_{\psi}} C_{\psi}^{2}\right)+\frac{\epsilon}{2}\left|\mu_{\tau_{k}}^{\prime}\right|(t)^{2}
\end{aligned}
$$

with $C_{V}:=\sup _{x \in \Omega}(|V(x)|+|\nabla V(x)|), C_{\psi}:=\sup _{(t, x) \in \mathbb{R} \times \Omega}\left(|\psi(t, x)|+\left|\nabla_{x} \psi(t, x)\right|\right), T_{\psi}>0$ such that $\psi(t, \cdot) \equiv 0$ for all $t \geq T_{\psi}$, and $C_{T_{\psi}}:=\sup \left\{\int_{\Omega} u_{\tau_{k}}(t, x) \mathrm{d} x: k \in \mathbb{N}, t \in\left(0, T_{\psi}\right)\right\}$ (which is finite by (4.23)), so that the limit inferior of $\left(\mathfrak{I}_{\tau_{k}, \psi}(t)+\epsilon\left|\mu_{\tau_{k}}^{\prime}\right|(t)^{2}\right)_{k \in \mathbb{N}}$ is indeed either $+\infty$ (if there is no bounded subsequence of $\left.\left(\left|\mu_{\tau_{k}}^{\prime}\right|(t)\right)_{k \in \mathbb{N}}\right)$ or the limit of some subsequence for which the corresponding subsequence of $\left(\left|\mu_{\tau_{k}}^{\prime}\right|(t)\right)_{k \in \mathbb{N}}$ is bounded. We may apply Fatou's lemma in order to obtain

$$
\begin{aligned}
& \liminf _{k \rightarrow \infty} \int_{0}^{\infty} \mathfrak{I}_{\tau_{k}, \psi}(t) \mathrm{d} t+\epsilon \Delta_{T_{\psi}} \geq \liminf _{k \rightarrow \infty} \int_{0}^{T_{\psi}}\left[\mathfrak{I}_{\tau_{k}, \psi}(t)+\epsilon\left|\mu_{\tau_{k}}^{\prime}\right|(t)^{2}\right] \mathrm{d} t \geq \int_{0}^{T_{\psi}} \liminf _{k \rightarrow \infty}\left[\mathfrak{I}_{\tau_{k}, \psi}(t)+\epsilon\left|\mu_{\tau_{k}}^{\prime}\right|(t)^{2}\right] \mathrm{d} t \\
\geq & \int_{0}^{T_{\psi}} \int_{\Omega}\left[\Lambda\left\langle\nabla L_{F}(u(t, x))+u(t, x) \nabla V(x), \nabla_{x} \psi(t, x)\right\rangle+\Sigma\left(\hat{L}_{F}(u(t, x))+V(x) u(t, x)\right) \psi(t, x)\right] \mathrm{d} x \mathrm{~d} t=\mathcal{I}_{F, V, \psi, u}
\end{aligned}
$$


with $\mathcal{I}_{F, V, \psi, u}$ defined as in (4.15) and $\Delta_{T_{\psi}}$ according to (4.25). This shows that

$$
\lim _{k \rightarrow \infty} \int_{0}^{\infty} \mathfrak{I}_{\tau_{k}, \psi}(t) \mathrm{d} t=\mathcal{I}_{F, V, \psi, u} \quad \text { for every } \psi \in \mathrm{C}_{\mathrm{c}}^{2}(\mathbb{R} \times \Omega)
$$

because we may let $\epsilon \downarrow 0$ and switch between $\psi$ and $-\psi$. The proof of (4.14) for $\psi \in \mathrm{C}_{\mathrm{c}}^{2}(\mathbb{R} \times \Omega)$ is complete.

Now, we include the no-flux boundary condition (1.22) in a weak form and prove (4.14) for all $\psi \in \mathrm{C}_{\mathrm{c}}^{2}\left(\mathbb{R} \times \mathbb{R}^{d}\right)$. The reason why we cannot just repeat the previous proof for $\psi \in \mathrm{C}_{\mathrm{c}}^{2}(\mathbb{R} \times \Omega)$ is that the derivation of (4.22) from Proposition 3.3, Assumption 4.7 and (4.21) will fail for general continuously differentiable functions $v$ with compact support in $\mathbb{R}^{d}$ (but not in $\Omega$ ), cf. Example 4.9 below. However, using the preceding results, a necessary condition of first order can still be obtained in this case. For $k \in \mathbb{N}, n \in \mathbb{N}$, let $\gamma_{\tau_{k}}^{n} \in \mathcal{M}(\bar{\Omega} \times \bar{\Omega})$ be optimal in the definition of $\mathrm{HK}_{\Lambda, \Sigma}\left(\mu_{\tau_{k}}^{n}, \mu_{\tau_{k}}^{n-1}\right)^{2}$ according to (1.10), with first marginal $\gamma_{\tau_{k}, 1}^{n} \ll \mu_{\tau_{k}}^{n}$ and second marginal $\gamma_{\tau_{k}, 2}^{n} \ll \mu_{\tau_{k}}^{n-1}$, and Lebesgue decompositions

$$
\mu_{\tau_{k}}^{n}=\rho_{\tau_{k}, 1}^{n} \gamma_{\tau_{k}, 1}^{n}+\left(\mu_{\tau_{k}}^{n}\right)^{\perp} \quad \text { and } \quad \mu_{\tau_{k}}^{n-1}=\rho_{\tau_{k}, 2}^{n} \gamma_{\tau_{k}, 2}^{n}+\left(\mu_{\tau_{k}}^{n-1}\right)^{\perp}
$$

cf. (3.11). By Corollary 3.4, Assumption 4.7, (4.21), the same arguments showing (4.22) and as $L_{F}\left(u_{\tau_{k}}^{n}\right) \in$ $\mathrm{W}^{1,1}(\Omega)$, we obtain the following necessary condition of first order

$$
\int_{\Omega}\left[\left\langle\nabla L_{F}\left(u_{\tau_{k}}^{n}\right), v\right\rangle+2 \hat{L}_{F}\left(u_{\tau_{k}}^{n}\right) R+(\langle\nabla V, v\rangle+2 V R) u_{\tau_{k}}^{n}\right] \mathrm{d} x=\frac{1}{\tau_{k}}\left(\mathfrak{F}_{\tau_{k}, n, v, R}-\frac{4}{\Sigma} \int_{\bar{\Omega}} R(x) \mathrm{d}\left(\mu_{\tau_{k}}^{n}\right)^{\perp}\right)
$$

for all $\mathrm{C}^{\infty}$-functions $v: \Omega \rightarrow \mathbb{R}^{d}$ with compact support in $\Omega$ and all bounded Borel measurable functions $R: \Omega \rightarrow \mathbb{R}$, where $\mathfrak{F}_{\tau_{k}, n, v, R}$ is defined as

$$
\begin{aligned}
& \frac{4}{\Sigma} \int_{\bar{\Omega} \times \bar{\Omega}}\left[-\rho_{\tau_{k}, 1}^{n}\left(x_{1}\right) R\left(x_{1}\right)+\sqrt{\rho_{\tau_{k}, 1}^{n}\left(x_{1}\right) \rho_{\tau_{k}, 2}^{n}\left(x_{2}\right)} R\left(x_{1}\right) \cos \left(\sqrt{\Sigma / 4 \Lambda}\left\|x_{1}-x_{2}\right\|\right)\right] \mathrm{d} \gamma_{\tau_{k}}^{n} \\
+ & \frac{4}{\Sigma} \int_{\bar{\Omega} \times \bar{\Omega}} \sqrt{\Sigma / 4 \Lambda \rho_{\tau_{k}, 1}^{n}\left(x_{1}\right) \rho_{\tau_{k}, 2}^{n}\left(x_{2}\right)}\left\langle S_{\Lambda, \Sigma}\left(x_{1}, x_{2}\right), v\left(x_{1}\right)\right\rangle \mathrm{d} \gamma_{\tau_{k}}^{n} .
\end{aligned}
$$

According to (1.10), Theorem 4.5 in [15] and Theorem 6.6 in [23], there exist a Borel function $\sigma_{\tau_{k}, 1}^{n}: \bar{\Omega} \rightarrow[0,+\infty)$ and a Borel optimal transport mapping $t_{\tau_{k}}^{n}: \bar{\Omega} \rightarrow \bar{\Omega}$ such that

$$
\gamma_{\tau_{k}, 1}^{n}=\sigma_{\tau_{k}, 1}^{n} \mu_{\tau_{k}}^{n}, \quad \gamma_{\tau_{k}}^{n}=\left(I \times t_{\tau_{k}}^{n}\right)_{\#} \gamma_{\tau_{k}, 1}^{n}=\left(I \times t_{\tau_{k}, 1}^{n}\right)_{\#}\left(\sigma_{\tau_{k}, 1}^{n} u_{\tau_{k}}^{n} \mathscr{L}^{d}\right) .
$$

Setting $R \equiv 0$ in (4.26) and applying (4.27), we obtain

$$
\begin{aligned}
& \int_{\Omega}\left\langle\nabla L_{F}\left(u_{\tau_{k}}^{n}(x)\right)+u_{\tau_{k}}^{n}(x) \nabla V(x), v(x)\right\rangle \mathrm{d} x \\
& =\frac{1}{\tau_{k}} \frac{4}{\Sigma} \int_{\Omega} \sqrt{\Sigma / 4 \Lambda \rho_{\tau_{k}, 1}^{n}(x) \rho_{\tau_{k}, 2}^{n}\left(t_{\tau_{k}}^{n}(x)\right)}\left\langle S_{\Lambda, \Sigma}\left(x, t_{\tau_{k}}^{n}(x)\right), v(x)\right\rangle \sigma_{\tau_{k}, 1}^{n}(x) u_{\tau_{k}}^{n}(x) \mathrm{d} x
\end{aligned}
$$

for all $\mathrm{C}^{\infty}$-functions $v: \Omega \rightarrow \mathbb{R}^{d}$ with compact support in $\Omega$. This shows that

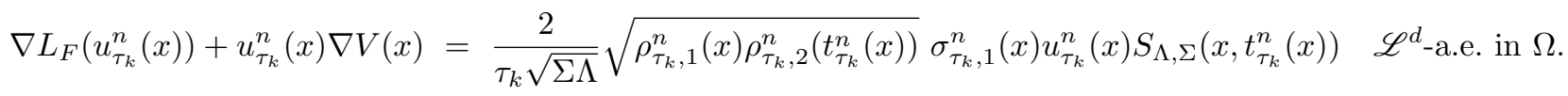


Consequently, (4.26) holds good for all bounded Borel measurable functions $v: \Omega \rightarrow \mathbb{R}^{d}$ and $R: \Omega \rightarrow \mathbb{R}$. Applying the proof of Corollary 3.4, Proposition 3.5 and the preceding arguments for $\psi \in \mathrm{C}_{\mathrm{c}}^{2}(\mathbb{R} \times \Omega)$, we obtain (4.14) for all $\psi \in \mathrm{C}_{\mathrm{c}}^{2}\left(\mathbb{R} \times \mathbb{R}^{d}\right)$. The proof of Theorem 4.4 is complete.

The following example shows that Assumption 4.7 does not imply such formula (4.17) for every continuously differentiable function $v: \mathbb{R}^{d} \rightarrow \mathbb{R}^{d}$ with compact support in $\mathbb{R}^{d}$.

Example 4.9. We can identify $\nu_{0}=u_{0} \mathscr{L}^{d} \in\{\mathcal{F}<+\infty\}$ with a nonnegative finite Radon measure $\nu_{0} \in \mathcal{M}\left(\mathbb{R}^{d}\right)$ by setting $u_{0} \equiv 0$ outside $\Omega$ and define the curve $h \mapsto \nu_{h} \in \mathcal{M}\left(\mathbb{R}^{d}\right)$ as in Assumption 4.7, for a continuously differentiable function $v: \mathbb{R}^{d} \rightarrow \mathbb{R}^{d}$ with compact support in $\mathbb{R}^{d}$ and a bounded Borel measurable function $R: \mathbb{R}^{d} \rightarrow \mathbb{R}^{d}$. Again, we obtain (4.19) which holds good on $\mathbb{R}^{d}$ for now. The measures $\nu_{h}$ can be restricted to measures $\nu_{h} \in \mathcal{M}(\bar{\Omega})$ and $\mathcal{F}\left(\nu_{h}\right):=\int_{\Omega} F\left(u_{h}(x)\right) \mathrm{d} x$.

Now, let $\Omega=(0,1), F(s):=s^{2}$ and $v: \mathbb{R} \rightarrow \mathbb{R}$ be a continuously differentiable function satisfying

$$
v(x) \begin{cases}=0 & \text { if }|x| \geq 2 \\ \in[-1,0) & \text { if }-2<x<1 \\ =-1 & \text { if } 0 \leq x<\frac{1}{2} \\ \geq 0 & \text { if } x \geq 1\end{cases}
$$

Then, for $h>0$ small enough, we have

$$
\frac{\mathcal{F}\left(\nu_{h}\right)-\mathcal{F}\left(\nu_{0}\right)}{h}=-\frac{1}{h} \int_{0}^{h} F\left(u_{0}(x)\right) \mathrm{d} x+\underbrace{\frac{1}{h} \int_{h}^{1}\left(F\left(\frac{u_{0}(x)(1+h R(x))^{2}}{1+h v^{\prime}(x)}\right)\left(1+h v^{\prime}(x)\right)-F\left(u_{0}(x)\right)\right) \mathrm{d} x}_{\rightarrow \int_{\Omega} u_{0}(x)^{2}\left(4 R(x)-v^{\prime}(x)\right) \mathrm{d} x \text { as } h \downarrow 0}
$$

and we note that the first term on the right-hand side cannot be controlled; if we take $u_{0}(x):=x^{-1 / 4}, x \in(0,1)$, then $\nu_{0}=u_{0} \mathscr{L}^{1} \in\{\mathcal{F}<+\infty\}$ and

$$
-\frac{1}{h} \int_{0}^{h} F\left(u_{0}(x)\right) \mathrm{d} x=-\frac{2 \sqrt{h}}{h} \rightarrow-\infty \quad \text { as } h \downarrow 0 .
$$

\subsection{Comments and outlook}

The discussion in Section 4.1 and Proposition 4.1 show that, under the assumptions of Theorem 4.4, every Generalized Minimizing Movement $\mu \in \operatorname{GMM}\left(\Phi ; \mu_{0}\right), \mu_{0} \in\{\mathcal{E}<+\infty\}$, associated with $\Phi$ and $\mathcal{E}$ as in (1.19) and (1.20), is locally absolutely continuous and satisfies the energy dissipation inequality (1.5), i.e.

$$
\mathcal{E}\left(\mu_{0}\right)-\mathcal{E}(\mu(t)) \geq \frac{1}{2} \int_{0}^{t}\left|\partial^{-} \mathcal{E}\right|(\mu(r))^{2} \mathrm{~d} r+\frac{1}{2} \int_{0}^{t}\left|\mu^{\prime}\right|(r)^{2} \mathrm{~d} r
$$

for all $t>0$.

Definition 4.10 (Absolutely continuous curves, relaxed slope). Let $(\mathscr{S}, d)$ be a complete metric space. We say that a curve $u:[0,+\infty) \rightarrow \mathscr{S}$ is locally absolutely continuous if there exists $m \in \mathrm{L}_{\text {loc }}^{1}(0,+\infty)$ such that

$$
d(u(s), u(t)) \leq \int_{s}^{t} m(r) \mathrm{d} r \quad \text { for all } 0 \leq s \leq t<+\infty .
$$


In this case, the limit

$$
\left|u^{\prime}\right|(t):=\lim _{s \rightarrow t} \frac{d(u(s), u(t))}{|s-t|}
$$

exists for $\mathscr{L}^{1}$-a.e. $t$, the function $t \mapsto\left|u^{\prime}\right|(t)$ belongs to $\mathrm{L}_{\text {loc }}^{1}(0,+\infty)$ and is called the metric derivative of $u$. The metric derivative is $\mathscr{L}^{1}$-a.e. the smallest admissible function $m$ in the definition above.

Let $\mathcal{E}: \mathscr{S} \rightarrow(-\infty,+\infty]$ be given. We define the local slope at $x \in\{\mathcal{E}<+\infty\}$ as

$$
|\partial \mathcal{E}|(x):=\limsup _{d(x, y) \rightarrow 0} \frac{(\mathcal{E}(x)-\mathcal{E}(y))^{+}}{d(x, y)}
$$

and the relaxed slope $\left|\partial^{-} \mathcal{E}\right|: \mathscr{S} \rightarrow[0,+\infty]$ of $\mathcal{E}$ as

$$
\left|\partial^{-} \mathcal{E}\right|(x):=\inf \left\{\liminf _{n \rightarrow \infty}|\partial \mathcal{E}|\left(x_{n}\right): d\left(x_{n}, x\right) \rightarrow 0, \sup _{n} \mathcal{E}\left(x_{n}\right)<+\infty\right\}
$$

We refer to ([2], Chaps. 1 and 2) for a detailed account of these and further definitions which are important in connection with the characterization of gradient flows in metric spaces by such energy dissipation (in)equality (cf. introductory part).

In this paper, we have focused on the fact that our Minimizing Movement scheme yields weak solutions to a class of reaction-diffusion equations. It will be worth studying the corresponding energy dissipation inequalities in more detail in a subsequent paper as they will provide additional information. The following example illustrates this.

Example 4.11. Let $d \in\{1,2\}$. For $F:[0,+\infty) \rightarrow \mathbb{R}, F(s):=-\sqrt{s}+s^{p}(p>1)$ and $V \equiv 0$, we define $\mathcal{E}$ and $\Phi$ as in (1.20) and (1.19). In this case, the functional $\mathcal{E}$ is geodesically convex on $\left(\mathcal{M}(\bar{\Omega}), \mathrm{H}_{\Lambda, \Sigma}\right)$ according to [21] and

$$
\left|\partial^{-} \mathcal{E}\right|(\mu)= \begin{cases}|\partial \mathcal{E}|(\mu) & \text { if } \mu \in\{\mathcal{E}<+\infty\} \\ +\infty & \text { else }\end{cases}
$$

by Cor. 2.4.10 in [2]. Obviously, $u \equiv 0$ is a solution to (4.14), (4.15). However, Theorem 4.4 (which is applicable in this example, $c f$. Ex. 4.8) will not yield this trivial solution, i.e.

$$
\eta_{0} \notin \operatorname{GMM}\left(\Phi ; \eta_{0}\right)
$$

(where $\eta_{0}$ denotes the null measure). Indeed, setting $\eta_{N}:=u_{N} \mathscr{L}^{d}, u_{N} \equiv \frac{1}{N}$ we can easily compute that

$$
\left|\partial^{-} \mathcal{E}\right|\left(\eta_{0}\right)=|\partial \mathcal{E}|\left(\eta_{0}\right) \geq \lim _{N \rightarrow \infty} \frac{-\mathcal{E}\left(\eta_{N}\right)}{\mathrm{HK}_{\Lambda, \Sigma}\left(\eta_{N}, \eta_{0}\right)}=\lim _{N \rightarrow \infty} \frac{\left(1 / \sqrt{N}-1 / N^{p}\right) \mathscr{L}^{d}(\Omega)}{2 / \sqrt{\Sigma} \sqrt{1 / N \mathscr{L}^{d}(\Omega)}}=\frac{\sqrt{\sum \mathscr{L}^{d}(\Omega)}}{2}>0
$$

which shows that the constant curve $\eta(t) \equiv \eta_{0}$ does not satisfy the corresponding energy dissipation inequality.

Our next comments concern different aspects of Theorem 4.4 which we have not considered so far for the sake of clear presentation. We may replace the initial datum $\mu_{0}=u_{0} \mathscr{L}^{d} \in\{\mathcal{E}<+\infty\}$ in the Minimizing Movement scheme (1.1) associated with (1.19), (1.20) by a sequence $\left(\mu_{\tau}^{0}\right)_{\tau}$ of measures $\mu_{\tau}^{0}=u_{\tau}^{0} \mathscr{L}^{d}$ satisfying $u_{\tau}^{0} \stackrel{L^{1}}{\rightarrow} u_{0}, \sup _{\tau} \mathcal{F}\left(\mu_{\tau}^{0}\right)<+\infty$, and still obtain the same results as in Theorem 4.4. Besides, we have left aside the 
possibility of adding an interaction energy functional to $\mathcal{E}$; it is not difficult to find appropriate assumptions in order to do so. We expect that our arguments will form the basis for a Minimizing Movement approach to scalar reaction-diffusion equations in other settings, too, e.g. if $X=\mathbb{R}^{d}$ or $X$ is a subset of a general separable Hilbert space or the energy functional is modified. We do not want to expound on how to adapt our assumptions and our proof for such cases, just give an example of suitable assumptions if $X=\mathbb{R}^{d}$.

Example 4.12. We suppose that $F:[0,+\infty) \rightarrow \mathbb{R}$ is continuous, strictly convex, differentiable in $(0,+\infty)$, has superlinear growth (4.7) and satisfies $F(0)=0, F(s) \geq-C_{F} s$ (for some $C_{F}>0$ ). Let $V: \mathbb{R}^{d} \rightarrow \mathbb{R}$ be locally Lipschitz continuous and let us suppose that $V \geq 0$ and $V(x) \rightarrow+\infty$ if $|x| \rightarrow+\infty$. We define $\mathcal{E}:=$ $\mathcal{F}+\mathcal{V}: \mathcal{M}\left(\mathbb{R}^{d}\right) \rightarrow(-\infty,+\infty]$ and $\Phi$ as in (1.20) and (1.19) with $\Omega$ replaced by $\mathbb{R}^{d}$, and we suppose that $\mathcal{F}$ satisfies a differentiability assumption which is like Assumption 4.7 (but with $\Omega$ and $\mathrm{L}^{1}\left(\Omega\right.$ ) replaced by $\mathbb{R}^{d}$ and $\mathrm{L}_{\text {loc }}^{1}\left(\mathbb{R}^{d}\right)$ respectively, and for continuously differentiable functions $v: \mathbb{R}^{d} \rightarrow \mathbb{R}^{d}$ and bounded Borel functions $R: \mathbb{R}^{d} \rightarrow \mathbb{R}, v$ and $R$ both with compact support in $\mathbb{R}^{d}$ ). Then similar arguments as in Section 4.2 will show that the associated Minimizing Movement approach yields weak solutions to the corresponding scalar reactiondiffusion equation on $\mathbb{R}^{d}$; the results are similar to those of Theorem 4.4. Note that the growth condition on $V$ makes an application of Dunford-Pettis-Theorem on $\mathbb{R}^{d}$ possible.

Acknowledgements. Giuseppe Savaré suggested this topic to me and I had the opportunity to discuss parts of it with him, Alexander Mielke and Martin Brokate; I would like to express my thanks to them. I gratefully acknowledge support from the Erwin Schrödinger International Institute for Mathematics and Physics (Vienna) during my participation in the programme "Optimal Transport".

\section{REFERENCES}

[1] F. Almgren, J.E. Taylor and L. Wang, Curvature-driven flows: a variational approach. SIAM J. Control Optim. 31 (1993) 387-438.

[2] L. Ambrosio, N. Gigli and G. Savaré, Gradient Flows in Metric Spaces and in the Space of Probability Measures. Lectures in mathematics. ETH Zürich, Birkhäuser (2005).

[3] A. Braides, Local Minimization, Variational Evolution and $\Gamma$-Convergence. Vol. 2094 of Lecture Notes in Mathematics. Springer (2012).

[4] L. Chizat and S. Di Marino, A tumor growth model of Hele-Shaw type as a gradient flow. ESAIM: COCV 26 (2020) 103.

[5] L. Chizat, G. Peyré, B. Schmitzer and F.-X. Vialard, An interpolating distance between optimal transport and Fisher-Rao metrics. Found. Comput. Math. 18 (2018) 1-44.

[6] L. Chizat, G. Peyré, B. Schmitzer and F.-X. Vialard, Unbalanced optimal transport: dynamic and Kantorovich formulations. J. Funct. Anal. 274 (2018) 3090-3123.

[7] E. De Giorgi, New problems on minimizing movements, in Boundary Value Problems for PDE and Applications, edited by C. Baiocchi and J.L. Lions. Masson (1993) 81-98.

[8] E. De Giorgi, A. Marino and M. Tosques, Problems of evolution in metric spaces and maximal decreasing curve. Atti Accad. Naz. Lincei Rend. Cl. Sci. Fis. Mat. Natur. 68 (1980) 180-187.

[9] M. Degiovanni, A. Marino and M. Tosques, Evolution equations with lack of convexity. Nonlinear Anal. 9 (1985) $1401-1443$.

[10] F. Fleißner, Gamma-convergence and relaxations for gradient flows in metric spaces: a minimizing movement approach. ESAIM: COCV 25 (2019) 28.

[11] F. Fleißner, A note on the differentiability of the Hellinger-Kantorovich distances. Preprint arXiv:2007.07225 (2020).

[12] F. Fleißner and G. Savaré, Reverse approximation of gradient flows as minimizing movements: a conjecture by De Giorgi. Annali della Scuola Normale di Pisa - Classe di Scienze 20 (2020) 677-720.

[13] I. Fonseca and G. Leoni, Modern Methods in the Calculus of Variations: Lp Spaces. Springer Science \& Business Media (2007).

[14] T.O. Gallouët and L. Monsaingeon, A JKO splitting scheme for Kantorovich-Fisher-Rao gradient flows. SIAM J. Math. Anal. 49 (2017) 1100-1130.

[15] W. Gangbo and R.J. McCann, The geometry of optimal transportation. Acta Math. 177 (1996) $113-161$.

[16] R. Jordan, D. Kinderlehrer and F. Otto, Free energy and the Fokker-Planck equation. Physica D 107 (1997) $265-271$.

[17] R. Jordan, D. Kinderlehrer and F. Otto, The variational formulation of the Fokker-Planck Equation. SIAM J. Math. Anal. 29 (1998) $1-17$.

[18] S. Kondratyev, L. Monsaingeon and D. Vorotnikov, A fitness-driven cross-diffusion system from population dynamics as a gradient flow. J. Differ. Equ. 261 (2016) 2784-2808.

[19] S. Kondratyev, L. Monsaingeon and D. Vorotnikov, et al., A new optimal transport distance on the space of finite Radon measures. Adv. Differ. Equ. 21 (2016) 1117-1164. 
[20] S. Kondratyev and D. Vorotnikov, Nonlinear Fokker-Planck equations with reaction as gradient flows of the free energy. $J$. Funct. Anal. 278 (2020) 108310.

[21] M. Liero, A. Mielke and G. Savaré, On geodesic $\lambda$-convexity with respect to the Hellinger-Kantorovich distance, in preparation.

[22] M. Liero, A. Mielke and G. Savaré, Optimal transport in competition with reaction: the Hellinger-Kantorovich distance and geodesic curves. SIAM J. Math. Anal. 48 (2016) 2869-2911.

[23] M. Liero, A. Mielke and G. Savaré, Optimal entropy-transport problems and a new Hellinger-Kantorovich distance between positive measures. Invent. Math. 211 (2018) 969-1117.

[24] A. Marino, C. Saccon and M. Tosques, Curves of maximal slope and parabolic variational inequalities on nonconvex constraints. Ann. Scuola Norm. Sup. Pisa Cl. Sci. 16 (1989) 281-330.

[25] A. Mielke, Differential, energetic, and metric formulations for rate-independent processes. Springer (2011).

[26] A. Mielke, A gradient structure for reaction-diffusion systems and for energy-drift-diffusion systems. Nonlinearity 24 (2011) 1329.

[27] A. Mielke and F. Rindler, Reverse approximation of energetic solutions to rate-independent processes. NoDEA Nonlinear Differ. Equ. Appl. 16 (2009) 17-40.

[28] A. Mielke, R. Rossi and G. Savaré, Balanced viscosity (BV) solutions to infinite-dimensional rate-independent systems. J. Eur. Math. Soc. 18 (2016) 2107-2165.

[29] F. Otto, The geometry of dissipative evolution equations: the porous medium equation. Commun. Partial Differ. Equ. 26 (2001) 101-174.

[30] C. Villani, Topics in optimal transportation. Vol. 58 of Graduate Studies in Mathematics. American Mathematical Society, Providence, RI (2003).

[31] C. Villani, Optimal transport. Old and new. Vol. 338 of Grundlehren der Mathematischen Wissenschaften. Springer-Verlag, Berlin (2009). 